OPEN ACCESS

Edited by:

Ive De Smet,

Flanders Institute for Biotechnology,

Belgium

Reviewed by:

Andrzej Miroslaw Pacak,

Adam Mickiewicz University

in Poznań, Poland

Isabel Diaz,

Universidad Politécnica de Madrid

(UPM), Spain

*Correspondence:

Verena Ibl

verena.ib/@univie.ac.at

tThese authors have contributed equally to this work.

Specialty section:

This article was submitted to

Plant Proteomics,

a section of the journal

Frontiers in Plant Science

Received: 21 January 2018

Accepted: 22 May 2018

Published: 13 June 2018

Citation:

Shabrangy A, Roustan V, Reipert S, Weidinger M, Roustan P-J, Stoger E, Weckwerth W and Ib/ V (2018) Using

RT-qPCR, Proteomics, and Microscopy to Unrave

the Spatio-Temporal Expression

and Subcellular Localization

of Hordoindolines Across

Development in Barley Endosperm.

Front. Plant Sci. 9:775.

doi: 10.3389/fpls.2018.00775

\section{Using RT-qPCR, Proteomics, and Microscopy to Unravel the Spatio-Temporal Expression and Subcellular Localization of Hordoindolines Across Development in Barley Endosperm}

\author{
Azita Shabrangy ${ }^{1 \dagger}$, Valentin Roustan ${ }^{1 \dagger}$, Siegfried Reipert ${ }^{2}$, Marieluise Weidinger ${ }^{2}$, \\ Pierre-Jean Roustan ${ }^{1}$, Eva Stoger ${ }^{3}$, Wolfram Weckwerth ${ }^{1,4}$ and Verena Ibl ${ }^{1 *}$ \\ ${ }^{1}$ Department of Ecogenomics and Systems Biology, University of Vienna, Vienna, Austria, ${ }^{2}$ Core Facility Cell Imaging and \\ Ultrastructure Research, University of Vienna, Vienna, Austria, ${ }^{3}$ Department of Applied Genetics and Cell Biology, University \\ of Natural Resources and Life Sciences, Vienna, Austria, ${ }^{4}$ Vienna Metabolomics Center, University of Vienna, Vienna, Austria
}

Hordeum vulgare (barley) hordoindolines (HINs), HINa, HINb1, and HINb2, are orthologous proteins of wheat puroindolines (PINs) that are small, basic, cysteine-rich seed-specific proteins and responsible for grain hardness. Grain hardness is, next to its protein content, a major quality trait. In barley, HINb is most highly expressed in the mid-stage developed endosperm and is associated with both major endosperm texture and grain hardness. However, data required to understand the spatio-temporal dynamics of HIN transcripts and HIN protein regulation during grain filling processes are missing. Using reverse transcription quantitative PCR (RT-qPCR) and proteomics, we analyzed HIN transcript and HIN protein abundance from whole seeds (WSs) at four [ 6 days after pollination (dap), 10, 12, and $\geq 20$ dap] as well as from aleurone, subaleurone, and starchy endosperm at two (12 and $\geq 20$ dap) developmental stages. At the WS level, results from RT-qPCR, proteomics, and western blot showed a continuous increase of HIN transcript and HIN protein abundance across these four developmental stages. Miroscopic studies revealed HIN localization mainly at the vacuolar membrane in the aleurone, at protein bodies (PBs) in subaleurone and at the periphery of starch granules in the starchy endosperm. Laser microdissetion (LMD) proteomic analyses identified $\mathrm{HINb} 2$ as the most prominent HIN protein in starchy endosperm at $\geq 20$ dap. Additionally, our quantification data revealed a poor correlation between transcript and protein levels of HINs in subaleurone during development. Here, we correlated data achieved by RT-qPCR, proteomics, and microscopy that reveal different expression and localization pattern of HINs in each layer during barley endosperm development. This indicates a contribution of each tissue to the regulation of HINs during grain filling. The effect of the high protein abundance of HINs in the starchy endosperm and their localization at the periphery of starch granules at late development stages at the 
cereal-based end-product quality is discussed. Understanding the spatio-temporal regulated HINs is essential to improve barley quality traits for high end-product quality, as hard texture of the barley grain is regulated by the ratio between $\mathrm{HINb} / \mathrm{HINa}$.

Keywords: barley endosperm, hordoindoline, spatio-temporal, laser microdissection, label-free shotgun proteomics, RT-qPCR, reference genes, grain hardness

\section{INTRODUCTION}

In barley, hordoindolines (HINs) are described as PIN ortholog proteins. Genetic studies have associated HIN proteins to both major endosperm texture and grain hardness (Darlington et al., 2001; Takahashi et al., 2010). The HIN gene family comprises the HINa, HINb1, and HINb2 genes (Darlington et al., 2001). HIN transcripts are endosperm specific; their mRNA level increased from 14 to 20 days after pollination (dap) in the endosperm and in the aleurone, and declined at 30 dap. Both HINa and $\mathrm{HINb}$ transcripts are more abundant in the endosperm than in the aleurone between 14 and 20 dap. However, no evidence was found that HINa plays a role in grain texture in barley as it was described for PINa (Darlington et al., 2001).

The protein family of puroindolines (PINs) has been affiliated with endosperm texture and grain hardness (Giroux and Morris, 1998). PINs are small, basic, cysteine-rich, seed-specific proteins (Blochet et al., 1993; Gautier et al., 1994). Both PINa and PINb are characterized by a tryptophan-rich domain, that is thought to be responsible for the interaction with neutral polar lipids (Blochet et al., 1993; Dubreil, 1997). PINa and PINb precursors are synthesized with a transit peptide and two additional cleavable propeptides at the $\mathrm{N}$ - and $\mathrm{C}$-terminal end. Originally, northern blot analyses of $P I N a$ and $P I N b$ during seed development showed an increase for both transcripts till 33 dap and a decrease at mature state (Gautier et al., 1994). PINa transcript abundance was always higher than the level of $P I N b$ transcripts during development and correlated with a higher protein content of PINa compared to PINb in wheat (Blochet et al., 1993). No PIN transcripts were detected in Triticum durum, indicating the specific expression of PINs in the common hexaploidy wheat Triticum aestivum (Dubreil, 1997; Dubreil et al., 1998). In T. aestivum, PINs are components of "friabilin," a complex mixture of neutral and basic polypeptides localized at the starch granule surface and are involved in endosperm softness (Greenwell and Schofield, 1986; Sourdille et al., 1996; Giroux and Morris, 1998). Transgenic rice expressing wheat PINa and $\mathrm{PINb}$ reduced seed hardness resulting in reduced starch damage and an increased amount of fine flour particles (Krishnamurthy and Giroux, 2001). Whereas PINa strongly binds to wheat phospholipids and glycolipids, PINb tightly binds only negatively charged phospholipids and forms loose lipoprotein complexes with glycolipids. According to this lipid binding, both PINs play a role in the formation and stability of bread dough foams (Dubreil, 1997). Additionally, PINs were reported to be involved in plant defense mechanisms against plant pathogens (Blochet et al., 1993) where in vitro assays show antifungal activity for all PINs (Dubreil et al., 1998). Additionally, transgenic rice plants expressing wheat $\mathrm{PINa}$ and $\mathrm{PINb}$ demonstrated an inhibition of fungal growth of Magnaporthe grisea and Rhizoctonia solani after infection (Krishnamurthy et al., 2001). Recent results further showed that PINs affect endosperm development and storage protein polymerization via the protein folding machinery and are thus responsible for the protein matrix formation (Lesage et al., 2011, 2012). In this context, the endosperm texture - and hence, PINa and PINb - affects the properties and quality of flour as the variation of kernel texture influences the milling energy, flour extraction rate, flour particle size, starch damage, pearling quality, malt extract yield, and dry-matter digestibility by ruminants (summarized in Pauly et al., 2013a,b). Microscopic analysis of PIN protein localizations was performed in wheat and oat by immunochemical confocal or by electron microscopy using different PIN antibodies (Gautier et al., 1994; Dubreil et al., 1998; Mohammadi et al., 2006). PINa and PINb localized in the protein matrix surrounding the starch granules in the starchy endosperm. Interestingly, PINa was also found in inclusions of aleurone grains in developing and in mature seeds (Gautier et al., 1994; Dubreil et al., 1998). PIN orthologs have been identified in oats and barley, but were absent in sorghum (Sorghum bicolor), maize (Zea mays), and rice (Oryza sativa; Li et al., 2008). In oat, tryptophanins (TRPs) were identified as PIN orthologs (Tanchak et al., 1998). Expression and localization studies showed that the amount of TRP proteins gradually increased in developing oat seeds and that they are localized at the surface of starch grains in the oat endosperm (Mohammadi et al., 2006).

The cereal caryopsis is composed of different cell layers with distinct, spatio-temporally regulated physiological and molecular mechanisms (Olsen, 2001, 2004). The developmental pathway of cereal endosperm consists of three distinct stages: syncytial, mitotic, and differentiation phase (Olsen, 2004). After differentiation, the fully developed endosperm can account for up to $75 \%$ of the seed weight and comprises four major cell types: an epidermal layer of aleurone cells surrounding the starchy endosperm cells, a basal layer of transfer cells, and the cells of the embryo-surrounding region (Olsen, 2001, 2004). The starchy endosperm thereby functions as storage site as it accumulates starch and seed storage proteins (SSPs; Olsen, 2004). The aleurone layer supports seed germination by mobilizing starch and SSP reserves in the starchy endosperm by releasing hydrolytic enzymes that help to degrade the stored nutrients in the endosperm (Olsen, 2004). Maize and wheat have one layer of aleurone cells, rice contains one to several layers, and barley has three layers of aleurone cells (Olsen, 2004). Whereas the persistent inner starchy endosperm cells are dead at the time of full maturation, the aleurone cells are viable and regulate the germination process (Young and Gallie, 2000).

Reverse transcription quantitative PCR (RT-qPCR) and mass spectrometry are high throughput, sensitive, and reproducible 
methods to quantify the relative abundance of mRNA and proteins, respectively. Normalization by RGs for RT-qPCR is critical and is still one of the most important challenges concerning this technique (Huggett et al., 2005; Ling and Salvaterra, 2011). However, the stability of RGs varies across different cell and tissue types, and even across developmental stages within the same cell or tissue type (Kozera and Rapacz, 2013). Thus, precise selection of RGs for a specific tissue and developmental stage is essential. Ten RGs were characterized for studying gene expression analyses in the developing barley caryopsis (spring cultivar Jersey; Ovesna et al., 2012). However, no characterized RGs are available to measure the relative abundance of mRNA transcripts both at the whole seed (WS) level and in different tissues during barley endosperm development in the barley cultivar Golden Promise (GP).

In this study, we used GP, to investigate the spatio-temporal expression alterations of HINs during endosperm development. The old spring two-row barley cultivar GP is characterized by a high frequency of transformants of immature embryos (Hiei et al., 2014) and is amenable to virus-induced (Holzberg et al., 2002) and RNA-induced gene silencing (Lee et al., 2012). Spatiotemporal microscopy studies describe the SSP pathways in the cultivar GP (Ibl and Stoger, 2014; Ibl et al., 2014).

We harvested GP seeds at four and at two development stages for WS and spatio-temporal RT-qPCR, proteomics, and microscopic studies, respectively, to study the spatio-temporal expression alterations and localization of HINs during barley GP endosperm development. In this context, RGs were validated and characterized to specifically normalize HIN transcripts in the WS as well as in distinct tissues during endosperm development. We could observe a constant increase of HIN transcripts and HIN proteins during grain development. Using laser microdissetion (LMD), we identified HINa and HINb2 to be most abundant in subaleurone and starchy endosperm at $\geq 20$ dap, respectively. In contrast, $\mathrm{HINb}$ transcript level was high in subaleurone at 12 dap, indicating a low correlation between transcripts and proteins in subaleurone. HINs are mainly localized to protein storage vacuoles (PSVs) in aleurone, at protein bodies (PBs) in subaleurone and at the periphery of starch granules in the starchy endosperm. In the context of the HIN trafficking pathway, the possible involvement of the endosomal sorting complex required for transport (ESCRT) is presented. Finally, we discuss the putative effects of HINs on the end-product quality.

\section{MATERIALS AND METHODS}

\section{Bioinformatics Concerning the RGs and HINs}

RG candidates were selected according to their performance (stability values, overall expression degrees) previously reported by Ovesna et al. (2012) and Faccioli et al. (2007). We designed new primers for almost all of those ten RGs [(SAM (S'adenosyl-L-methionine)) ELF (elongation factor 1alpha), GAP (glyceraldehyde-3-phosphate dehydrogenase), GRP (glycine-rich protein), HSP70 (Heat shock $70 \mathrm{kD}$ protein), ARF (ADP-ribosylation factor), FBPA (fructosebisphosphatealdolase),
HSP90 (heat shock protein 90), UBI (ubiquitin gene), ACPIII (acyl carrier protein III)] using Primer3 v.0.4.0 (Untergasser et al., 2007; Koressaar et al., 2018) from gene sequences available in the EMBL database resulting in small PCR products (Supplementary Table S1). Primer sequences were analyzed using BLAST at IPK Gatersleben homepage ${ }^{1}$ searching for full-length cDNA entries. Additionally, the specific PCR sequences and/or fulllength cDNA entries from each RG from the Unigene homepage were analyzed by BLAST using the UniProt platform ${ }^{2}$ searching for the corresponding protein.

We used the HINs sequence accession numbers and corresponding primers as previously described (Terasawa et al., 2012). The specificity of the HINa and HINb PCR products was confirmed by sequencing.

\section{Plant Material}

Barley wild-type variety GP was cultivated as described in (Ibl and Stoger, 2014). We chose four ear development stages for harvesting according to Ibl et al. (2014) and Peukert et al. (2014): one timepoint at prestorage phase (including transition phase) and three timepoints within the storage phase (Peukert et al., 2014). These timepoints include the most dramatic changes of the endomembrane system that is involved in SSP trafficking (Ibl et al., 2014). As the caryopses of the barley ear are heterogeneous in their development at certain developmental stages, we ended up in the following seed development stages: 6-8, 10, 12-18, and $\geq 20$ dap (Supplementary Figure S1). RT-qPCR as well as proteomic studies were performed on WS harvested at ear development stage $6-8,10,12-18$, and $\geq 20$ dap as well as on different cell layers isolated by LMD at $12-18$ and $\geq 20$ dap. For spatio-temporal analyses, seeds were harvested from $\pm 20 \%$ of the middle of the ear. For the proteomics studies, all seeds from the whole ear were taken to increase the total protein amount. Within this manuscript, we used " 6 dap," "10 dap," "12 dap," and " $\geq 20$ dap" to describe the stages $6-8,10,12-18$, and $\geq 20$ dap.

\section{Tissue Preparation for Laser Microdissection}

For LMD microscopy, caryopses between $\pm 20 \%$ from the middle of the ear were taken. Embryos of caryopses were dissected and sections from the central region of the grain were excised using a razor blade. They were transferred to a silicon mold (Plano, Wetzlar, Germany), cryogel (Plano, Wetzlar, Germany) was added, and samples were frozen in liquid nitrogen and stored at $-80^{\circ} \mathrm{C}$. The mold including the grain was fixed to a sample plate, and series of $20 \mu \mathrm{m}$ sections were cut and immediately mounted on SuperFrost ${ }^{\circledR}$ PLUS slides (Thermo Scientific, Karlsruhe, Germany), which were stored in a Falcon tube containing silica gel to absorb moisture. After $30 \mathrm{~min}$, these dry cryo sections were used for LMD experiments.

\section{Laser Microdissection}

The Leica ${ }^{\circledR}$ laser microdissecting microscope (LMD6500) with LMD Software was used for microdissection. The $355 \mathrm{~nm}$ laser

\footnotetext{
${ }^{1}$ http://webblast.ipk-gatersleben.de/barley_ibsc/

${ }^{2}$ http://www.uniprot.org/
} 
was set to isolate distinct tissues from the sectioned materials: the aleurone, subaleurone, and starchy endosperm were separated from the surrounding tissue by defining a closed cutting line, and the cut region was removed by gravity into a lid of a precooled PCR tube. Typically, aleurone and subaleurone were represented by 30 sections, in total equivalent to $2,100,000 \mu \mathrm{m}^{2}$ of surface area. Similarly, the starchy endosperm was represented by $\sim 16$ sections, equivalent in total to $6,300,000 \mu \mathrm{m}^{2}$. Three independent preparations were processed per cell type for subsequent RTqPCR.

\section{RNA Extraction and cDNA Synthesis}

Barley seeds at $6,10,12$, and $\geq 20$ dap were harvested for subsequent gene and protein expression analyses. All samples were stored at $-80^{\circ} \mathrm{C}$ until further processing. Total RNA was extracted from the WS as described in Li and Trick (2005). Total RNA from the LMD sections was extracted with a Picopure RNA isolation kit (Molecular Devices, Sunnyvale, CA, United States). We applied gene expression analysis by two step RT-qPCR according to the Minimum Information for Publication of Quantitative Real-Time PCR Experiments (MIQE) guidelines (Bustin et al., 2009). RNA concentration was measured at $260 \mathrm{~nm}$ and purity of RNA assessed by $\mathrm{OD}_{260} / \mathrm{OD}_{280}$ ratio using a UV spectrophotometer (NanoDrop Technologies, United States; Supplementary Figures S2, S3). RNA integrity of RNA isolated from WS as well as from different endosperm cell layers was assessed by a microfluidic capillary gel electrophoresis applying the Experion ${ }^{\mathrm{TM}}$ system (Experion ${ }^{\mathrm{TM}}$ RNA HighSens Analysis Kit, Bio-Rad Laboratories, United States) with Experion software 3.2P. The quality of RNA index (QRI) was between 4.7 (color indicator: yellow: possibly acceptable quality) and 9.7 (color indicator: green: acceptable quality) even though we are aware that plants have a further rRNA peak derived from 25S RNA. Selected RNA samples were stored at $-80^{\circ} \mathrm{C}$ prior to use. DNA was digested from total RNA (10 ng/ $\mu \mathrm{L})$ with RNase-Free DNase Set (Qiagen, Germany). Prior to reverse transcription, samples were held at $42^{\circ} \mathrm{C}$ for 2 min with gDNA Wipeout Buffer (Qiagen, Germany). Reverse transcription was performed on 5 ng RNA in a final volume of $20 \mu \mathrm{L}$, using Quantitect Reverse Transcription Kit (Qiagen, Germany). Samples were incubated for $25 \mathrm{~min}$ at $42^{\circ} \mathrm{C}, 3 \mathrm{~min}$ at $95^{\circ} \mathrm{C}$, and cooled at $4^{\circ} \mathrm{C}$ or stored at $-80^{\circ} \mathrm{C}$. cDNA synthesis and RT-qPCR were performed with three biological replicates. We used a pipetting robot (Qiagen, Germany) and RT-qPCR was performed with the Corbett RotorGene Q (Australian TM Corbett Research Pty Ltd.) using the QuantiFast TM SYBR Green RT-PCR Kit (Qiagen, Germany). At least three pipetting replicates were for done for RT-qPCR. The PCR cycling conditions were set as follows: PCR initiation activation step for $5 \mathrm{~min}$ at $95^{\circ} \mathrm{C}$, denaturation for $5 \mathrm{~s}$ at $95^{\circ} \mathrm{C}$ and combined annealing extension for $10 \mathrm{~s}$ at $60^{\circ} \mathrm{C}$. The cycle number was 40 . To verify the consistency of the amplicon, a melting point analysis was performed.

\section{RT-qPCR and Data Analyses}

Besides bioinformatic analyses, the specificity of the primers was tested by PCR on cDNAs transcribed from RNAs extracted from WSs at 12 dap (Supplementary Figure S2). Quality analyses for all RNAs, RT-qPCRs' parameters for each run and normalization was carried out for all WS and LMD analyses as well as for RGs and HINs studies and was performed as the following (Supplementary Figures S2-S5): performing RT-qPCR, a single peak was found for the melting curve for all 10 candidate RGs at each time point (data not shown). Slope, $R^{2}$ value (squared correlation coefficient), and the PCR efficiency parameters were used to validate the PCR reaction of each primer pair. The amplification factor (AF) was calculated for each gene depending on the slope. Knowing that an optimal RT-qPCR reaction should have a slope of -3.32 (optimal range between -3.58 and -3.10 ), an efficiency of $100 \%$ and an $R^{2}$ value of $>0.99$, we evaluated only the PCR runs closest to these values. To determine the relative quantities $(Q)$, we calculated the mean Ct value for each biological replicate of four developmental stages for the WS analyses and of two developmental stages for LMD analyses. For the WS as well as for LMD analyses, $Q=E^{\Delta C T}$, where $E$ is the efficiency of the gene amplification and $\triangle C T$ is the sample with the lowest $\mathrm{Ct}$ in the dataset minus the $\mathrm{Ct}$ value of the gene of interest and thus normalized to the lowest $\mathrm{Ct}$ value of the gene. The coefficient of variation $(\mathrm{CV})$ was calculated for each candidate $\mathrm{RG}$. The $\mathrm{CV}$ is the ratio of the standard deviation to the mean (average) of all $\mathrm{Ct}$ values of three biological replicates of a candidate RG. Thus, four representing values were used for the analysis using the statistical algorithms tool geNorm to calculate the expression stability value $(M)$ for each candidate RG for the WS (Vandesompele et al., 2002; Andersen et al., 2004). For LMD studies, three values for 12 and $\geq 20$ dap and six values for aleurone, subaleurone, and starchy endosperm were employed for the geNorm analyses. $M$ describes the average of the pairwise variation of each particular gene with all other candidate reference genes. The lower the $M$ value is, the more stably expressed the gene is. Additionally, the CV was calculated for each RG candidate to evaluate its expression stability.

For the normalization studies of $H I N$ transcripts, we used the following RGs (Supplementary Table S2): ARF, FBPA, and SAM for WS; GAP, GRP, and UBI for LMD at 12 dap; HSP90, ACPIIII, and $A R F$ for LMD at $\geq 20$ dap; HSP70, HSP90, GRP, ELF, UBI, and FBPA for A (12 and $\geq 20$ dap); SAM, GRP, HSP70, ARF, $H S P 90, F B P A, E L F$, and $U B I$ for SA (12 and $\geq 20$ dap); and ELF, $F B P A$, and $U B I$ for SE (12 and $\geq 20$ dap). Normalization was calculated as described (Vandesompele et al., 2002). For statistical analyses, we performed a Student's $t$-test [two-tailed distribution, two-sample unequal variance (heteroscedastic)] by the software Microsoft Excel.

\section{Sample Preparation for Proteomics Analyses, MS Measurement, and Data Analysis}

Total proteins were extracted from barley seeds harvested at $6,10,12$, and $\geq 20$ dap as well as from LMD sections from seeds harvested at 12 and $\geq 20$ dap in three biological replicates (Supplementary Figure S1) following an adapted protocol from (Roustan et al., 2017). A phenol-phase protocol extracted proteins. Samples were dissolved in a urea buffer and protein concentration was measured with a Bradford Assay prior to 
trypsin digestion. Following over-night digestion, peptides were desalted with C18 solid phase extraction (SPE) tips (Agilent Technologies, Santa Clara, CA, United States). After SPE, the corresponding eluates were dried in a vacuum concentrator. Peptide pellets were dissolved at a protein concentration equivalent to $0.1 \mu \mathrm{g} / \mu \mathrm{L}$ in $5 \%(\mathrm{v} / \mathrm{v}) \mathrm{ACN}$ and $0.1 \%(\mathrm{v} / \mathrm{v})$ formic acid (FA); $0.5 \mu \mathrm{g}$ of the mixture was separated on an EASYSpray PepMap RSLC $75 \mu \mathrm{m} \times 50 \mathrm{~cm}$ column (Thermo Fisher Scientific Inc., Waltham, United States). Peptides were eluted using a 240 min linear gradient from 2 to $40 \%$ of mobile phase B (mobile phase A: $0.1 \%[\mathrm{v} / \mathrm{v}] \mathrm{FA}$ in water; mobile phase B: $0.1 \%[\mathrm{v} / \mathrm{v}] \mathrm{FA}$ in $90 \%[\mathrm{v} / \mathrm{v}] \mathrm{ACN})$ with $300 \mathrm{~nL} / \mathrm{min}$ flow rate generated with an UltiMate 3000 RSLCnano system. For the LMD samples, peptides were eluted with a $145 \mathrm{~min}$ linear gradient with the same mobile phase and percentage as for the WS proteomics. The peptides were measured with a LTQ-Orbitrap Elite (Thermo) using the following mass analyzer settings: ion transfer capillary temperature $275^{\circ} \mathrm{C}$, full scan range 350-1800 $\mathrm{m} / z$, FTMS resolution 120,000. Each FTMS full scan was followed by up to 10 data-dependent (DDA) CID tandem mass spectra (MS/MS spectra) in the linear triple quadrupole (LTQ) mass analyzer. Dynamic exclusion was enabled using list size $500 \mathrm{~m} / z$ values with exclusion width $\pm 10 \mathrm{ppm}$ for $60 \mathrm{~s}$. Charge state screening was enabled and unassigned and +1 charged ions were excluded from MS/MS acquisitions. For injection control, automatic gain control (AGC) for full scan acquisition in the Orbitrap was set to $5 \times 105$ ion population, the maximum injection time ( $\max$ IT) was set to $200 \mathrm{~ms}$. Orbitrap online calibration using internal lock mass calibration on $\mathrm{m} / z 371.10123$ from polydimethylcyclosiloxane was used. Multistage activation was enabled with neural losses of 24.49, 32.66, 48.999, 97.97, 195.94, and 293.91 Da for the 10 most intense precursor ions. Prediction of ion injection time was enabled and the trap was set to gather $5 \times 103$ ions for up to $50 \mathrm{~ms}$. Raw files were processed with MaxQuant $1.5^{3}$ and Andromeda search algorithm (Cox and Mann, 2008; Cox et al., 2011) on the Hordeum vulgare sp. vulgare Uniprot database (124,660 protein entries). Peptides identification was performed using the following settings: Mass tolerance for precursor was set to $5 \mathrm{ppm}$ and for fragment masses up to $0.8 \mathrm{Da}$. The maximum FDR was set to $0.01 \%$. Three missed cleavages were allowed. The dynamic modifications allowed were: methionine oxidation (M) and protein N-terminal acetylation. Carbamidomethyl (C) was allowed as fixed modification. Labelfree quantification (LFQ) was performed based on at least two peptides per protein across the developmental stages and was internally normalized by MaxQuant (Cox et al., 2014). Quantification was calculated at peptide level. Further data processing (including one-way ANOVA) was performed with the Perseus 1.5 software (Tyanova et al., 2016). Annotated MS/MS spectra were visualized with MaxQuant (Tyanova et al., 2015). For statistical analyses, we performed a Student's $t$-test [two-tailed distribution, two-sample unequal variance (heteroscedastic)] by the software Microsoft Excel. The mass spectrometry proteomics data have been deposited to the

${ }^{3}$ http://www.maxquant.org
ProteomeXchange Consortium (Deutsch et al., 2017) via the PRIDE (Vizcaino et al., 2016) partner repository with the dataset identifier PXD009708 and PXD009722.

\section{Western Blot (WB) Protocol for HIN Detection}

Developing seeds of barley were harvested at 6,12 , and $\geq 20$ dap. One hundred milligrams of each fresh grain were squashed in $2 \mathrm{~mL}$ of reducing agent $(25 \mathrm{mM}$ Tris- $\mathrm{HCl} \mathrm{pH} \mathrm{7.8,} \mathrm{1.6 \%}$ SDS, $100 \mathrm{mM} \mathrm{DTT}$ ) using mortar and pestle on ice. The homogenate was centrifuged at $6800 \mathrm{~g}$ for $15 \mathrm{~min}$ at $4^{\circ} \mathrm{C}$ and the supernatant was transferred into a fresh microtube; $20 \mu \mathrm{L}$ of SDS-PAGE sample buffer [containing $250 \mathrm{mM}$ Tris- $\mathrm{HCl} \mathrm{pH}$ $6.8,10 \%(\mathrm{w} / \mathrm{v})$ SDS, $0.5 \%(\mathrm{w} / \mathrm{v})$ bromophenol blue and $50 \%$ (v/v) glycerol] was added to $80 \mu \mathrm{L}$ of each sample, boiled for $5 \mathrm{~min}$ in microtubes, and subsequently cooled at room temperature. They were loaded in 5\% stacking polyacrylamide gel and fractionated in 15\% resolving gel for $2 \mathrm{~h}$ at a constant current of $25 \mathrm{~mA}$ under denaturing condition using a BioRad mini-gel electrophoresis unit. The starting voltage was $52 \mathrm{~V}$ and final voltage $124 \mathrm{~V}$. Electrode buffer was $25 \mathrm{mM}$ Tris-base $\mathrm{pH} 8.8,200 \mathrm{mM}$ glycine, and $0.1 \%$ (w/v) SDS. After electrophoresis, the gel was presoaked in the blotting buffer [48 mM Tris-base $\mathrm{pH} 8.3,39 \mathrm{mM}$ glycine, 20\% (v/v) methanol], together with $3 \mathrm{~mm}$ Whatman filter paper and nitrocellulose membrane for $30 \mathrm{~min}$. Proteins were transformed from gel to nitrocellulose membrane using the Bio-Rad semi-dry transblotter at constant $18 \mathrm{~V}$ for $30 \mathrm{~min}$. Following the transfer, the nitrocellulose membrane was blocked in $5 \%(\mathrm{w} / \mathrm{v})$ non-fat powdered milk $(1 \mathrm{~h})$ prepared in a phosphate buffered saline including $0.1 \%$ Tween 20 (PBS-T pH 7.4). Immunoblots were incubated in 1:2000 dilution of anti-PINa/b rabbit antiserum prepared in PBS-T buffer for $2 \mathrm{~h}$ at room temperature or at $4^{\circ} \mathrm{C}$ shaking overnight, followed by washing three times in PBS-T, 5 min each. The second antibody was anti-rabbit IgG-alkaline phosphatase conjugate that was diluted 1:5000 and incubated for $1 \mathrm{~h}$. Signal detection was performed by ready-to-use reagents (Bio-Rad).

\section{Fixation, Semi-thin Sectioning, and Immunofluorescence}

At least three randomly selected seeds were harvested from the mid-section of the ear at 12 and $\geq 20$ dap (Supplementary Figure S1). They were tangentially and vertically cut into one to $2 \mathrm{~mm}^{2}$ pieces and fixed in $2.5 \%$ paraformaldehyde and $0.25 \%$ glutaraldehyde in $0.1 \mathrm{M}$ phosphate buffer (PB) $\mathrm{pH} 7.4$ at $4^{\circ} \mathrm{C}$ overnight. The chemically fixed samples underwent lowtemperature dehydration and infiltration with methacrylic resin, Lowicryl HM20 (Polysciences, Warrington, PA, United States), by applying the progressive lowering of temperature technique (Carlemalm et al., 1985). After washing in PBS, the samples were dehydrated in a series of ethanol (30\% ethanol for $30 \mathrm{~min}$ on ice; $50 \%$ ethanol for $45 \mathrm{~min}$, two times $70 \%$ ethanol for $30 \mathrm{~min}$ each, $95 \%$ ethanol for $45 \mathrm{~min}$, and two times $100 \%$ ethanol for $30 \mathrm{~min}$ each, on salt ice at $\left.-20^{\circ} \mathrm{C}\right)$. Subsequently, samples were infiltrated with HM20 
at $-20^{\circ} \mathrm{C}(1 / 3$ volume $\mathrm{HM} 20$ and $2 / 3$ volume ethanol for $1 \mathrm{~h}, 2 / 3$ volume HM20 and $1 / 3$ volume ethanol for $2 \mathrm{~h}$, pure HM20 for $1 \mathrm{~h}$ ). The infiltration with pure resin was continued for further $6 \mathrm{~h}$ at $-40^{\circ} \mathrm{C}$ in an automated freeze substitution unit (AFS2, LEICA Microsystems, Austria). For UV polymerization, samples were transferred into PCR tubes, Multiply ${ }^{\circledR} 0.2 \mathrm{~mL}$ (Sarstedt) that were attached to spider covers (LEICA Microsystems, Austria) as described previously (Reipert and Wiche, 2008). UV-polymerization was performed for $36 \mathrm{~h}$ at $-40^{\circ} \mathrm{C}$.

Semithin sections $(1.5 \mu \mathrm{m})$ of the seeds were cut by an ultramicrotome LEICA EM UC7 (Wetzlar, Germany) and a semi-diamond knife (DIATOME Ltd., Switzerland), collected on a glass slide and dried at $40^{\circ} \mathrm{C}$. Seed sections were rinsed in PBS for $20 \mathrm{~min}$ and blocked with 5\% BSA in $0.1 \mathrm{M}$ $\mathrm{PB}$, for $15 \mathrm{~min}$ at room temperature. The sections were incubated with rabbit polyclonal anti-PINa/b (diluted 1:50 in $0.1 \mathrm{M} \mathrm{PB}$ ) followed by three times washing by $0.5 \%$ Tween in $0.1 \mathrm{M}$ PB. The secondary antibody Alexa $488^{\circledR}$ was diluted 1:30 in $0.1 \mathrm{M} \mathrm{PB}$ and incubated for $1 \mathrm{~h}$ at room temperature. Sections were washed in $0.1 \mathrm{M} \mathrm{PB}$ and distilled water three times for $10 \mathrm{~min}$ each, followed by air drying. At least two slides containing three sections each were analyzed and images were quantified with ImageJ. For statistical analyses, we performed a Student's $t$-test ([two-tailed distribution, two-sample unequal variance (heteroscedastic)] by the software Microsoft Excel.

\section{RESULTS}

\section{Detection and Quantification of HIN Transcripts in WS During Barley Endosperm Development}

In order to explore the dynamics of HIN transcripts during endosperm development, we analyzed first the levels of $\mathrm{HINa}$ and both $\mathrm{HINb}$ transcripts in developing WS. cDNA alignment analyses of $H I N a, H I N b 1$, and HINb2 showed that the DNA sequence $H I N a$ is different to $H I N b 1$ and $H I N b 2$, but the cDNA sequences of $H I N b 1$ and $H I N b 2$ are very similar (Figure 1A). Thus, specific primers could only be designed for HINa and HINb resulting in small PCR products (Figure 1A, Supplementary Figure S6, and Supplementary Table S3). According to our geNorm results, we used the three most stable RGs $A R F, F B P A$, and $S A M$ to normalize HIN transcripts in WS during endosperm development at $6,10,12$, and $\geq 20$ dap. No significant changes of the abundance of HIN transcripts could be observed in WSs during development, even though both HIN transcripts showed a trend to increase during development, especially the HINa transcripts (Figure 1B). These results correlate to data previously shown where HIN transcripts increased during late development stages (Darlington et al., 2001). To confirm the stability of the selected RGs, we normalized the HIN transcripts to the lowest stable RG GRP. The bar chart shows a decrease for both HIN transcripts between 6 and $\geq 20$ dap and subsequently no reliable normalization results (Supplementary Figure S7).

\section{Detection and Quantification of HIN Proteins in WS During Barley Endosperm Development}

Quantitative proteomics across barley endosperm developmental stages was performed. In total, 1029 proteins were quantified across the four studied stages of barley grain filling in all samples (Supplementary Table S4). At the protein level, MS1 precursors were used to specifically quantify $\mathrm{HINa}$ as well as HINb1 and HINb2. In total, two peptides were common to HINb1 and HINb2 and seven, four, and six peptides were unique for $\mathrm{HINb} 2, \mathrm{HINb} 1$, and $\mathrm{HINa}$, respectively (Figure 2A and Supplementary Figure S8A). As well, some of the peptides were found in the LMD-based proteomics approach as indicated in Supplementary Figure S8A. Mass spectrometry analysis specifically identified proteotypic peptides corresponding to the tryptophan-rich domain of HINa, HINb1, and HINb2 (Figure 2B and Supplementary Figure S8B) by the presence of three trypsintarget residues $(\mathrm{R} / \mathrm{K})$. In barley, the tryptophan-rich domain of HINb2, 61 - KDFPVTWPTKWWKG - 74, presented exactly the same amino acid sequence as PINb in wheat (Wall et al., 2010). Quantification of the HIN proteins at the WS level highlights a constant significant increase of the HIN abundance from 6 to $\geq 20$ dap (Figures 3A-C). Between 6 and $\geq 20$ dap, HINb2 became the most abundant protein (Figure 3D). More specifically, HINb2 was approximatively 1.5 times more abundant than HINa and 3.4 times more abundant than HINb1 at $\geq 20$ dap (Figure 3D). Those proportions are in line with the HIN proportion found previously in mature seeds (Mahalingam, 2017). To confirm the LC-MS data, we performed a western blot (WB) of protein extracts of 6,12 , and $\geq 20$ dap. HINs are barley homologs of wheat PIN and oat TRP proteins that show high similarity at the level of amino acid sequence (Supplementary Figure S8B) confirmed also by MS/MS spectrum (Figure 2B). Therefore, we used anti-PINa/b antisera (kindly provided by Illimar Altosaar) that recognize both A- and B-isomers of the wheat PIN homolog (Mohammadi et al., 2006). Whereas hardly any band could be detected at 6 and 12 dap, anti-PINa/b recognized two bands at $\geq 20$ dap (Supplementary Figure S8C). As the full protein of $\mathrm{HINa}$ has $16.4 \mathrm{kDa}$ and HINb1/b2 $16.1 / 16 \mathrm{kDa}$, we concluded that the upper band represents $\mathrm{HINa}$ and $\mathrm{HINb}$ proteins. The lowest band described a $13 \mathrm{kDa}$ protein, which represents the mature protein without signal peptide and propeptides. This indicates a specific recognition of barley HINs by anti-PINa/b, as the WB showed no signal when incubated by the pre-immune-serum (Supplementary Figure S8C). Thus, HINs are most accumulated at $\geq 20$ dap where HINb2 describes the most abundant protein, followed by HINa and finally HINb1.

\section{HINs Are Mainly Localized at PSVs in Aleurone, at PBs in Subaleurone, and at the Periphery of Starch Granules in the Starchy Endosperm}

As HINs start to accumulate at 12 dap and increased to $\geq 20$ dap, these two timepoints were chosen to explore the localization of HINs in developing barley grain. Seeds were harvested at 12 and 
A DQ269851_HINa AY644090- HinbI : : ATGAAGGCCTTCTTCCTCGTGGGTCTGCTTGCTITGGTAG DQ862334_Hinb2
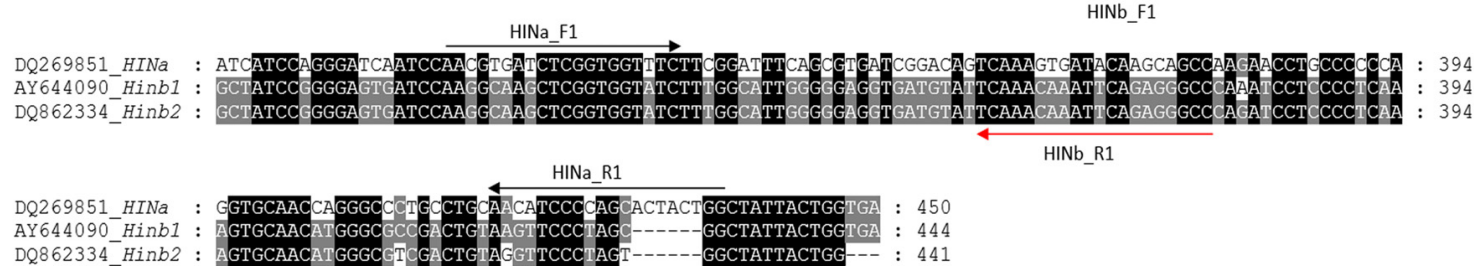

B

HINs WS 6 - $\geq 20$ dap (ARF, FBPA, SAM)

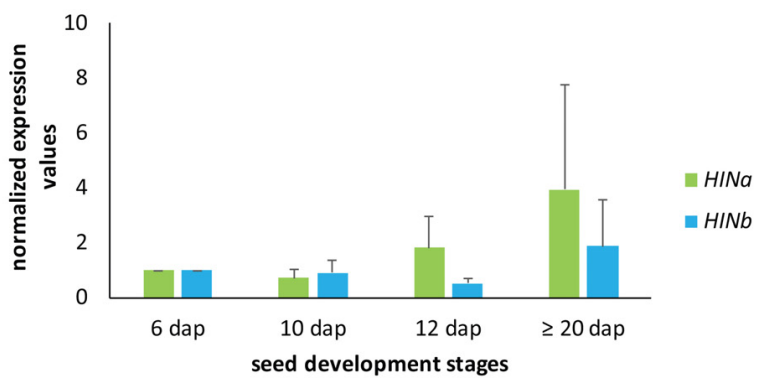

FIGURE 1 | Gene alignment and quantification of HINa and HINb transcripts in WS during barley endosperm development. (A) cDNA alignment of HINa, Hinb1, and HINb2 performed by MEGA7.0.21 (Kumar et al., 2016) and visualized by GenDoc (Nicholas and Nicholas, 1997). Conserved percentage is shown as the following: black $100 \%$, dark gray $80 \%$. (B) Bar graph describes the average over three biological replicates of the normalized transcripts from HINa and HINb at 6, 10, 12, and $\geq 20$ dap with the most stable RGs (ARF, FBPA, and SAM). Bars represent standard deviation. For statistical analyses, we performed a Student's $t$-test. Note the trend of all HIN transcripts to increase between 6 and $\geq 20$ dap.

$\geq 20$ dap; fixed and semi-thin sections $(1.5 \mu \mathrm{m})$ were prepared for microscopic studies. We used anti-PINa/b antibodies for a spatio-temporal visualization of both HIN isoforms ( $a$ and b) by immunofluorescence. We could observe a strong signal in the subaleurone and starchy endosperm at 12 dap as well as at $\geq 20$ dap (Figure $4 \mathbf{A}$ ), whereas no signal was observed in the negative control (Supplementary Figure S9). Using a higher magnification, we could identify a prominent fluorescent signal at the typically shaped PBs (Ibl et al., 2014) and at the periphery of starch granules in subaleurone at 12 and $\geq 20$ dap (Figure 4B). The signal at the PBs in subaleurone appeared to remain constant, whereas the amount of intensively labeled PBs increased in the starchy endosperm between 12 and $\geq 20$ dap (Figure 4B). The signal at the periphery of starch granules was detectable in the starchy endosperm at 12 dap and became stronger at $\geq 20$ dap. Interestingly, a weak signal was observed at the PSV membrane in the aleurone tissue at $\geq 20$ dap (Figure $4 \mathbf{B}$ ). We quantified the fluorescent signal of several sections in aleurone, subaleurone, and starchy endosperm at 12 and $\geq 20$ dap by ImageJ (Supplementary Figure S10A; Schindelin et al., 2012; Schneider et al., 2012). At 12 dap, the signal in subaleurone and starchy endosperm was 2.7 and 4.8 times stronger than in aleurone, respectively (Supplementary Figure S10B). The signal at $\geq 20$ dap was again stronger in starchy endosperm (two times more) and in subaleurone (1.2 times more) than in aleurone. Within one tissue, the strongest increase of the fluorescent signal between 12 and $\geq 20$ dap could be observed in aleurone (three times more), followed by the increase of the signal in the starchy endosperm (1.4 times more). These results strongly point to a spatio-temporal increase of the HIN protein abundance in barley endosperm. Additionally, we observed subcellular localization alterations of HINs depending on the cell layer and development stage. Especially the high HIN abundance in the starchy endosperm at 12 and $\geq 20$ dap is accompanied by an accumulation of HINs at the periphery of starch granules.

\section{Quantification of Spatio-Temporal HIN Proteins and HIN Transcripts in Developing Barley Endosperm}

To get further insight into the proportion of HIN proteins and $H I N$ transcripts in aleurone, subaleurone, and starchy endosperm 


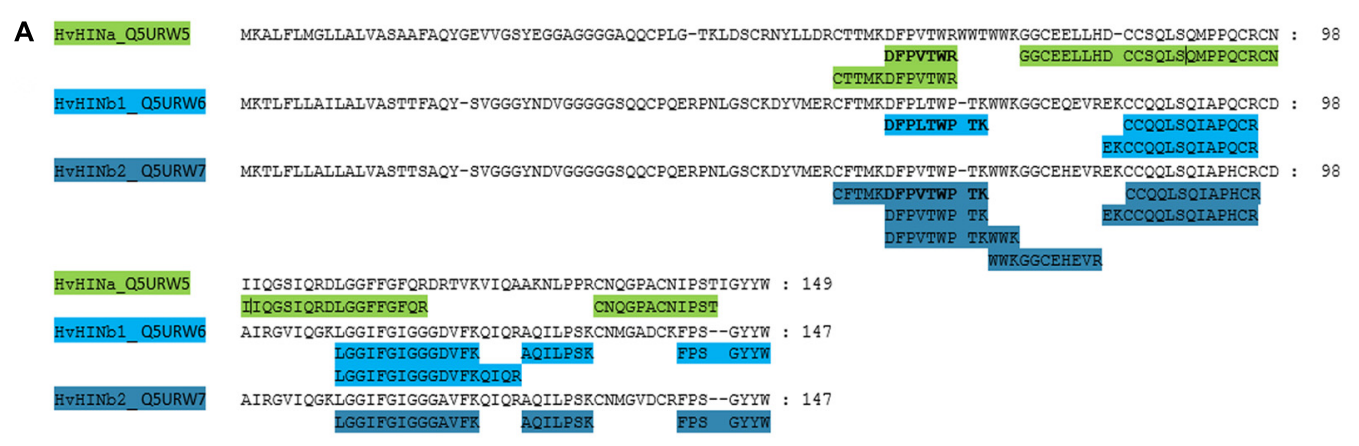

B

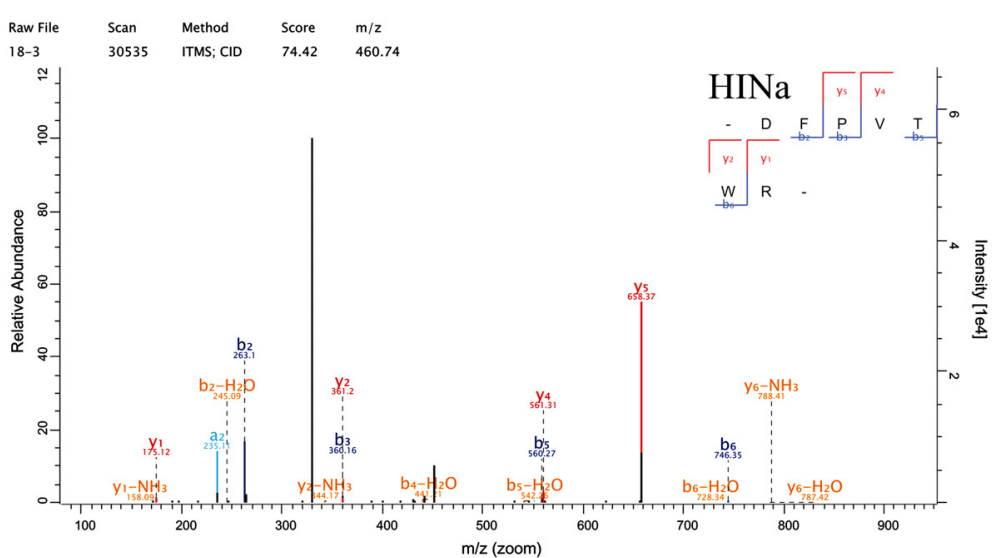

$\begin{array}{lllll}\text { Raw File } & \text { Scan } & \text { Method } & \text { Score } & \mathrm{m} / \mathrm{z} \\ 10-1 & 37503 & \text { ITMS; CID } & 84.21 & 552.79\end{array}$

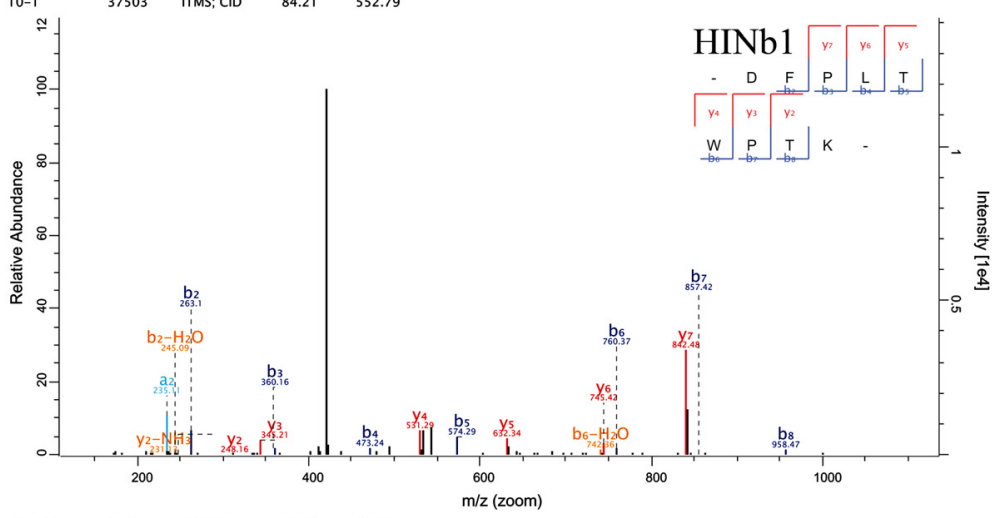

$\begin{array}{lllll}\text { Raw File } & \text { Scan } & \text { Method } & \text { Score } & \mathrm{m} / \mathrm{z} \\ 10-2 & 34267 & \text { ITMS; CID } & 117.89 & 545.78\end{array}$

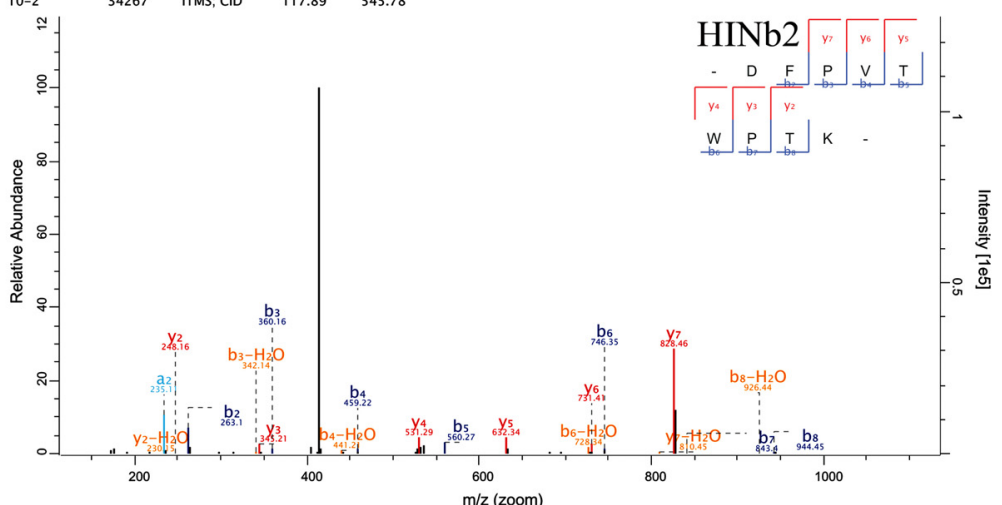

FIGURE 2 | Proteomic identification of HIN proteins. (A) Identified peptides in the proteomic approach have been aligned with HINa, HINb1, and HINb2 protein sequence. (B) MS/MS spectra of the identified tryptophan-rich peptides of $\mathrm{HINa}, \mathrm{HINb1}$, and $\mathrm{HINb2}$. 

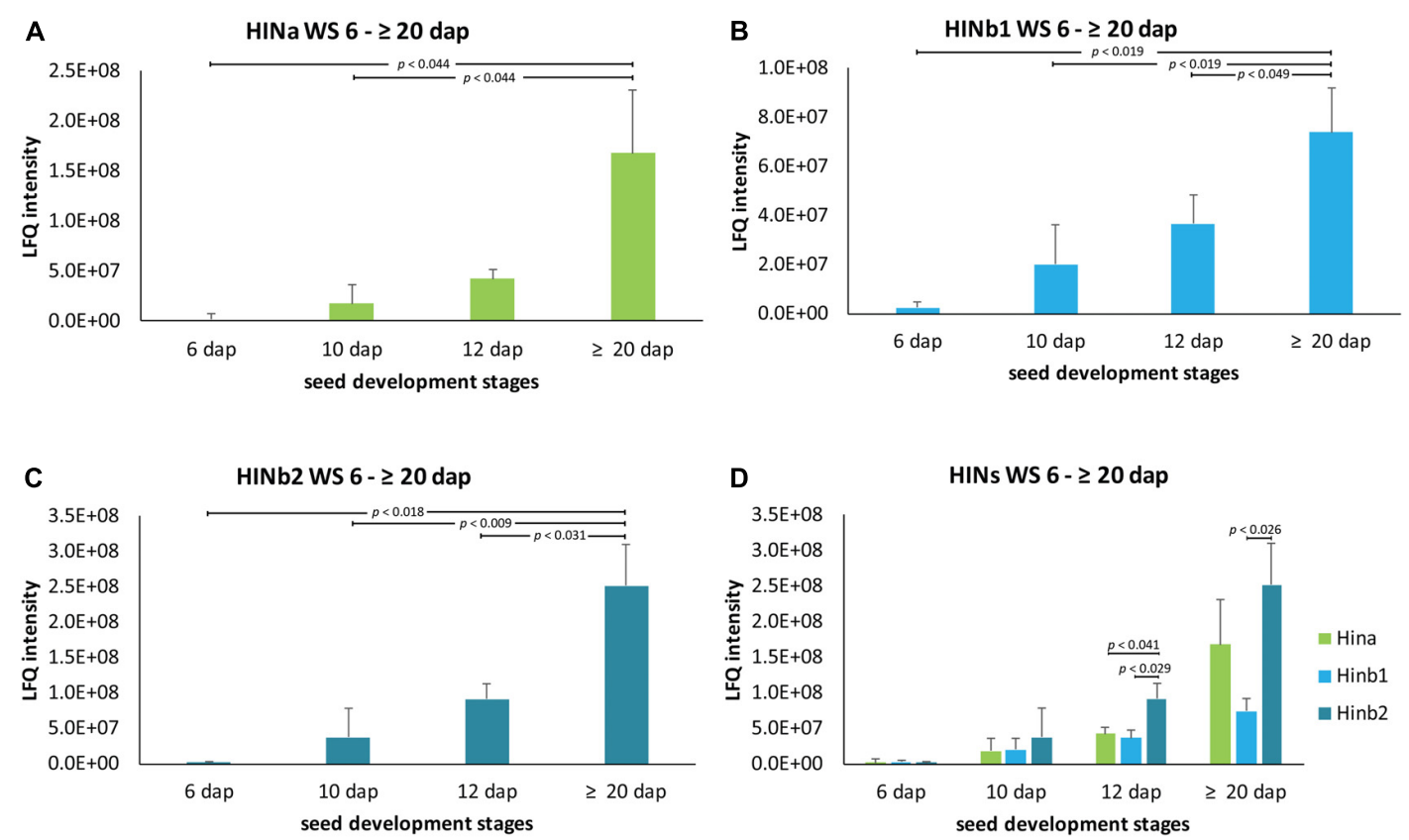

FIGURE 3 | LFQ quantification of HIN proteins in WS between 6 and $\geq 20$ dap based on ion precursor intensity. (A) HINa, (B) HINb1, and (C) HINb2 protein abundance in WS between 6 and $\geq 20$ dap. (D) Comparative analysis of HINa, HINb1, and HINb2 abundance. For statistical analyses, we performed a Student's $t$-test. LFQ intensities of proteins were averaged over three biological replicates. Bars represent standard deviation. Note the indicated $p$-values.

during the grain filling process, we used LMD for sampling three different tissues at two timepoints (12 and $\geq 20$ dap) for subsequent quantitative shotgun proteomics and RT-qPCR. As indicated by the immunolabeling of the HIN proteins, there is an important dynamic range between the starchy endosperm and the aleurone and subaleurone layer. Such a dynamic range affects the peptide detection, since in mass spectrometry, there is no amplification of the signal such as in RT-qPCR. This effect was supported by the LMD-based shotgun proteomics data. Indeed, most of the peptides' identification and quantification were performed in the starchy endosperm tissue (Supplementary Table S4). More precisely, the three HIN proteins were well detectable in the starchy endosperm at both 12 and $\geq 20$ dap (with a consistent quantification of at least two peptides per proteins). The identification rate is less in the aleurone and subaleurone layer: only one peptide specific to HINb2 was detectable in the aleurone layer at $\geq 20$ dap. This is in line with the detected increase of HIN content in the aleurone cell layer between 12 and $\geq 20$ dap by immunofluorescence (Supplementary Figure S10). The increase of HIN proteins in aleurone from 12 to $\geq 20$ dap is further supported by the HINa and HINb1 peptides detection in only one sample in aleurone tissue at $\geq 20$ dap (Supplementary Table S4). Surprisingly, a single peptide belonging to HINa was detected in one subaleurone sample at $\geq 20$ dap. In order to approximate a distribution pattern of the HINs, we averaged the intensity of the peptides and plotted them in a spatio-temporal pattern (Figure 5 and Supplementary Table S4). Whereas HINa and HINb2 showed the trend to increase in the starchy endosperm between 12 and $\geq 20$ dap (Figures $5 \mathbf{A}, \mathbf{C}$ ), HINb1 increased significantly (Figure 5B). HINb2 is the most abundant HIN protein in starchy endosperm at $\geq 20$ dap, followed by HINa and finally HINb (Figure 5D). These results confirmed the major contribution of the starchy endosperm to the total seed HIN content (Figure 5D). Interestingly, the proportions between the HIN protein abundances are possibly conserved in the starchy endosperm between 12 and $\geq 20$ dap as the same distribution of HIN proteins could be detected.

To get a complete spatio-temporal RNA expression overview of the HIN transcripts, we quantified changes in the abundance of HIN transcripts in aleurone, subaleurone, and starchy endosperm during development by using our characterized RGs for these tissues (Figures 6A-E). At 12 dap, HINb transcripts were significantly more abundant in subaleurone and starchy endosperm compared to aleurone (Figure 6A). The most significant changes of $\mathrm{HINa}$ transcripts could be observed in subaleurone (Figure 6D), where they increased significantly between 12 and $\geq 20$ dap (Figure 6D). Interestingly, HINb transcripts are more abundant than $\mathrm{HINa}$ ones in subaleurone at 12 dap (Figure 6D), whereas only HINa was detectable in subaleurone at $\geq 20$ dap.

These data suggest a spatio-temporal regulation of both transcription and translation of HINs where the subaleurone showed a poor correlation between HIN transcripts and proteins.

\section{DISCUSSION}

Barley grain ranks fourth in cereal production worldwide but is the second largest cereal crop within the European Union (19.5 

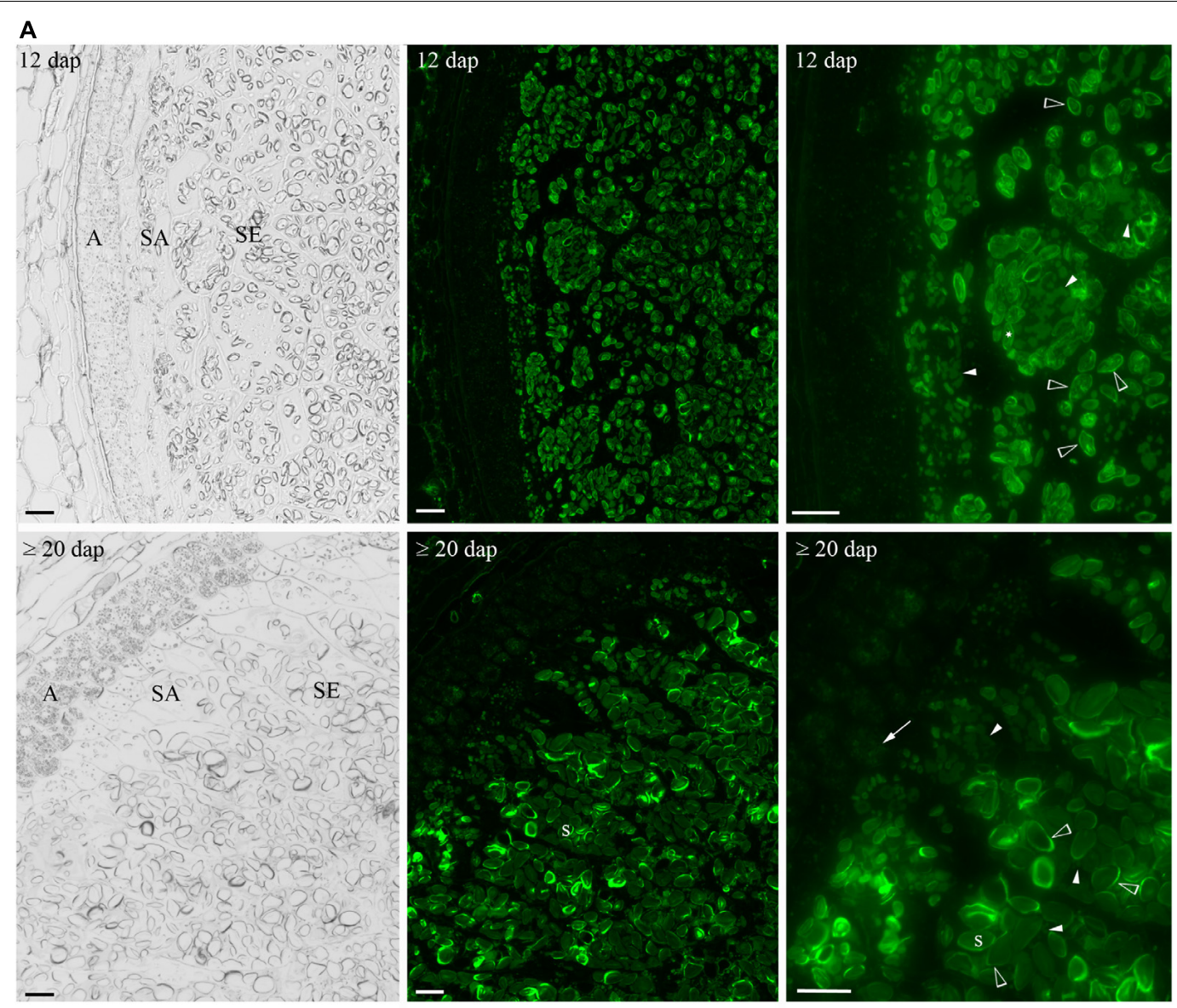

B
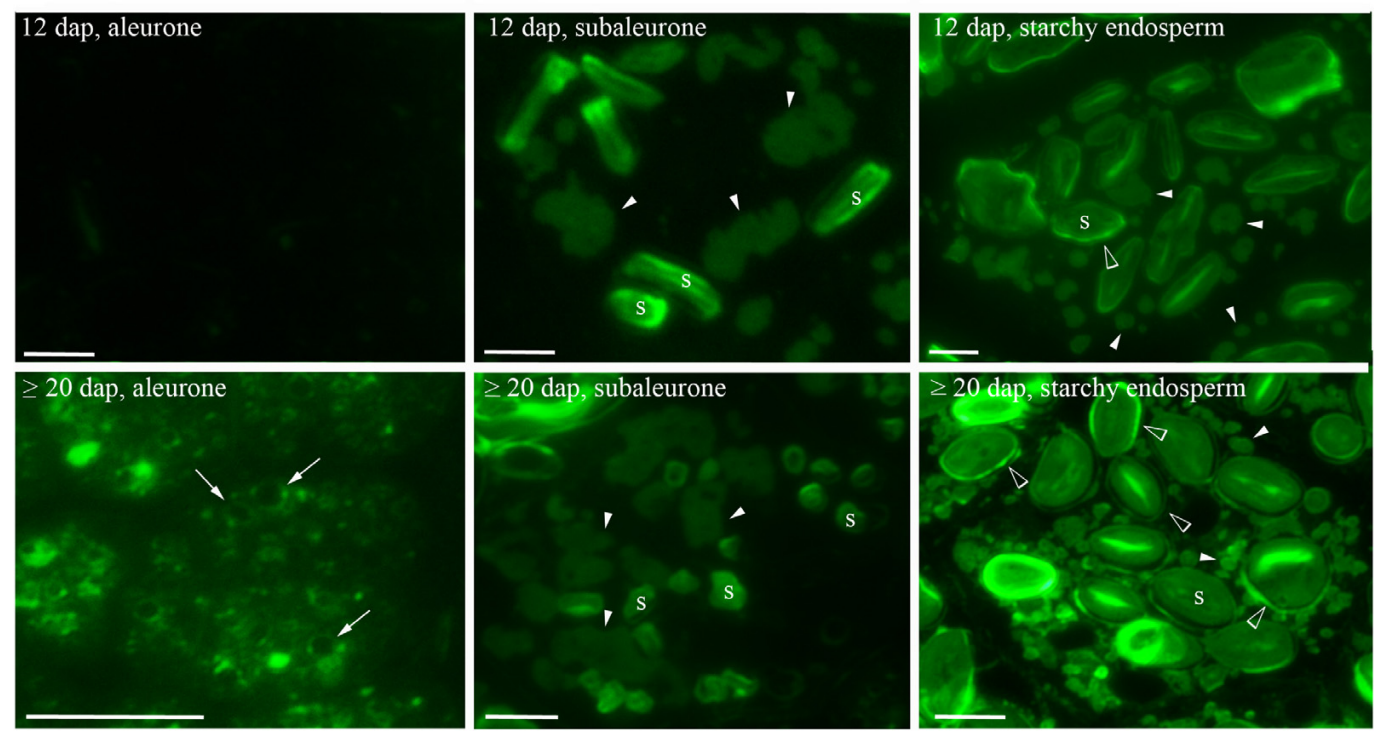

FIGURE 4 | Spatio-temporal localization of HINs during barley endosperm development. (A) Anti-PINa/b labeling of HINs at 12 and $\geq 20$ dap. Note the layers of aleurone (A), subaleurone (SA), and the starchy endosperm (SE) in the bright field channel. At 12 dap, no signal of HINs could be detected in aleurone, whereas a faint signal could be detected in $A$ (arrows) at $\geq 20$ dap. At 12 and $\geq 20$ dap, anti-PINa/b strongly labels protein bodies (PBs) in SA and in SE, respectively (arrowheads). (B) Close-up of a cell representing each tissue. The signal at PBs appears to be constant in SA between 12 and $\geq 20$ dap (arrowheads). Note the signal of HINs at the vacuolar membrane (arrows) in the $A$ at $\geq 20$ dap and the strong signal at the periphery of starch granules in the SE at $\geq 20$ dap (open arrowheads). Labeled PBs show reduced sizes in SE at 12 and $\geq 20$ dap (arrowheads). Scale $=100 \mu \mathrm{m}$. s indicates starch granules. 


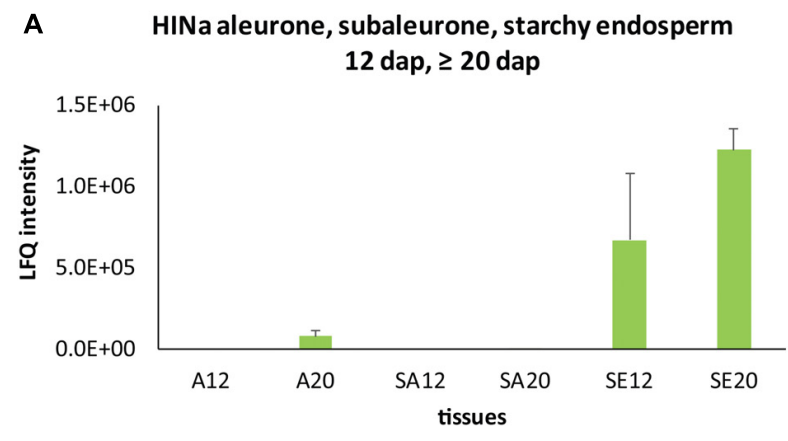

C

HINb2 aleurone, subaleurone, starchy endosperm 12 dap, $\geq 20$ dap

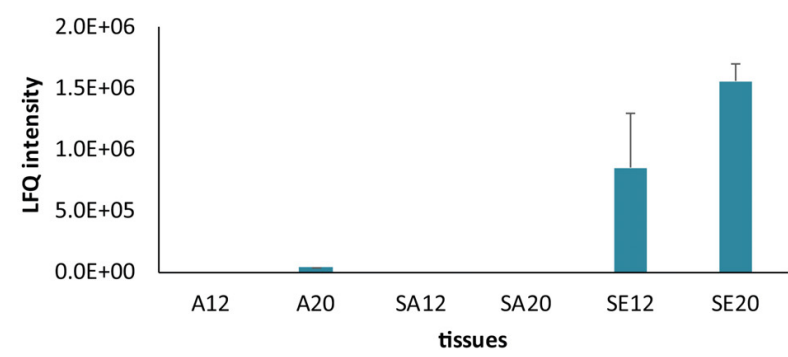

B

HINb1 aleurone, subaleurone, starchy endosperm 12 dap, $\geq 20$ dap

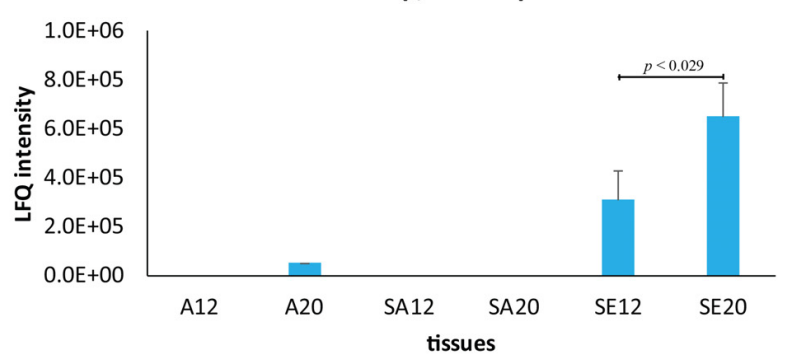

D

HINs aleurone, subaleurone, starchy endosperm 12 dap, $\geq 20$ dap

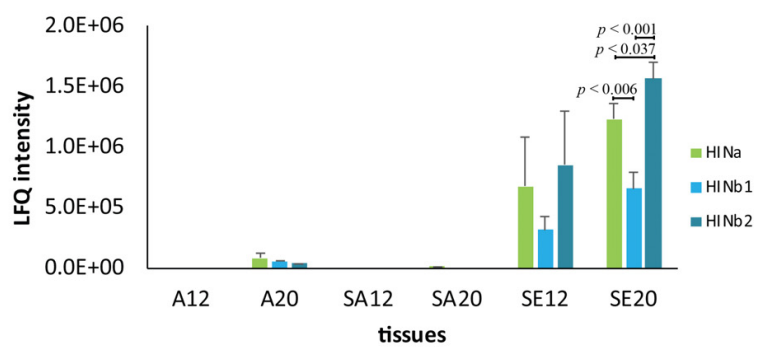

FIGURE 5 | Spatio-temporal HIN protein LFQ quantification in developing barley endosperm. Protein quantification of (A) HINa, (B) HINb1, and (C) HINb2 in aleurone (A), subaleurone (SA), and in starchy endosperm (SE) at 12 and $\geq 20$ dap. (D) Overview of HIN proteins in A, SA, and SE at 12 and $\geq 20$ dap. For statistical analyses, we performed a Student's $t$-test. LFQ intensities of proteins were averaged over three biological replicates. Bars represent standard deviation. Note the indicated $p$-values.

$\%$ of total cereal production ${ }^{4}$ ). Besides its importance as food and feed source, the most frequent use of barley is for malting purposes for the brewing industry (Gupta et al., 2010). Grain texture of barley has a huge impact on the malting performance, and soft barley cultivars have better malting quality (Brennan et al., 1996; Psota et al., 2007; Gupta et al., 2010). The endospermspecific PIN orthologs HINa, HINb1, and HINb2 are associated with both major endosperm texture and grain hardness in barley.

In wheat, PINa and PINb proteins are associated with grain hardness and endosperm/kernel texture, the leading characteristics of common "bread" wheat. In addition, PINs are important players concerning the foam formation and stability of beer as the addition of PIN proteins to degas could protect beer foam against lipid-induced destabilization (Bamforth, 1985; Clark et al., 1994). PINs are furthermore involved in antimicrobial activities and therefore considered for developing applications (reviewed in Bhave and Morris, 2008). While the spatio-temporal regulation of PINs expression is quite well studied in wheat, little is known about their homologous proteins in barley. Therefore, it is essential to understand the molecular regulation of HIN proteins during barley endosperm development. For this, a detailed analysis of the spatio-temporal expression of HINs during barley endosperm development as well as their subcellular localization is necessary. In this regard, we conduct three main experiments: first, we identified RGs

${ }^{4}$ http://ec.europa.eu/eurostat/ for reliable quantification studies of HIN transcripts during WS endosperm development as well as in three different tissues at two different time points. Second, we performed proteomics to quantify the protein abundances of HINa, HINb1, and HINb2 during WS endosperm development as well as in each cell layer at different timepoints. Third, we studied the localization of HIN proteins during barley endosperm development. The combination of RT-qPCR, proteomics, and microscopic analyses of HINs not only gives insight into the spatio-temporal regulation during barley development, but also strengthens our data as the results are correlating. This is illustrated by the data-matrix heat map in Figure 7 that shows a high abundance of all HIN transcripts and HIN proteins in starchy endosperm at $\geq 20$ dap.

Having developed a new panel of RGs for spatio-temporal normalization studies in barley GP, we quantified the transcripts of $\mathrm{HINa}$ and $\mathrm{HINb}$ in the WS at 6,10,12, and $\geq 20$ dap. We could not separate HINb1 from HINb2 by RT-qPCR as no specific primer could be designed due to their overlapping CDNA sequence. Both $H I N a$ and $H I N b$ transcripts showed the trend to increase at $\geq 20$ dap. Using LC-MS and WB analyses, we could detect an increase of the protein abundance for both HINa and $\mathrm{HINb}$ from 6 to $\geq 20$ dap, indicating a correlation between the amount of HIN transcript and protein abundance. Although the HINbs are very similar proteins, the resolution of LC-MS allowed the identification of unique peptides for both HINb1 and HINb2: out of the 19 identified peptides, 17 were unique peptides 
A HINs aleurone, subaleurone, starchy endosperm 12 dap (GAP, GRP, UBI)

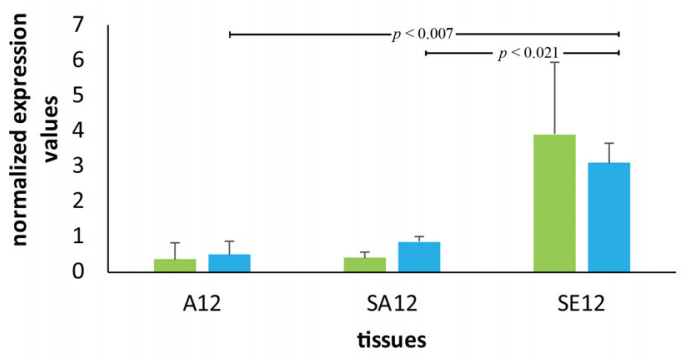

C

HINs aleurone 12, $\geq 20$ dap (HSP70, HSP90, GRP, ELF, UBI, FBPA)

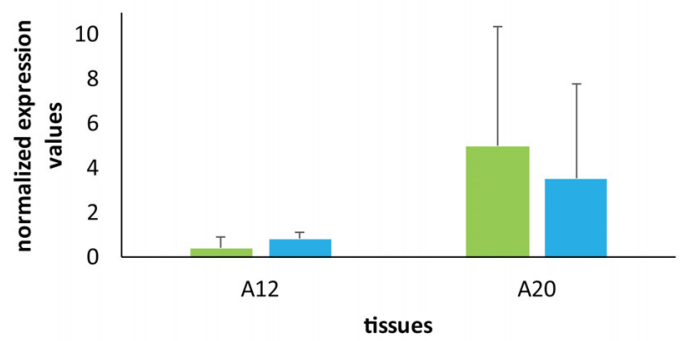

E

HINs starchy endosperm 12, $\geq 20$ dap (ELF, FBPA, UBI)

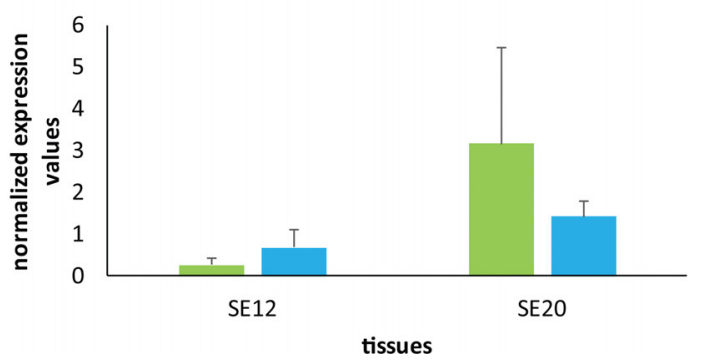

B
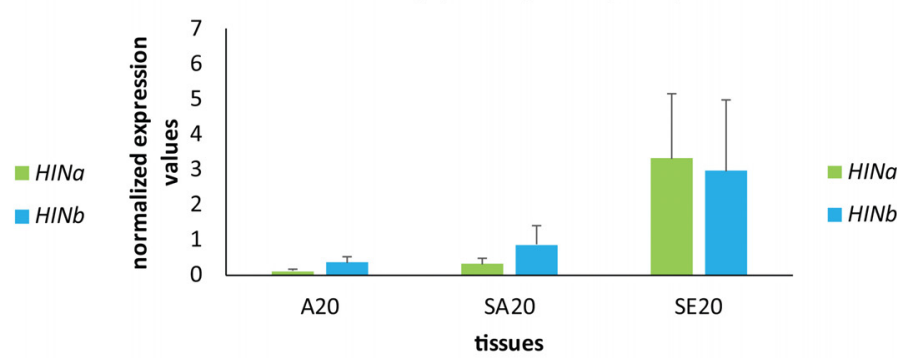

D

HINs subaleurone 12, $\geq 20$ dap (SAM, GRP, HSP70, ARF, HSP90, FBPA, ELF, UBI)

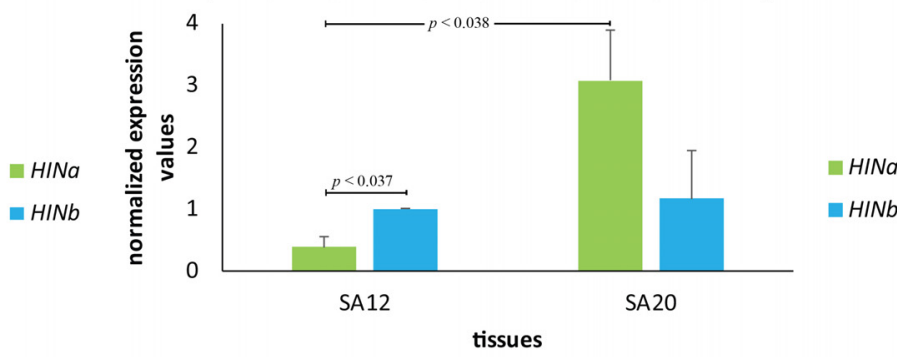

IINa

- HINb

FIGURE 6 | Spatio-temporal HIN transcripts quantification in developing barley endosperm. Bar graph describes the average over three biological replicates of the normalized transcripts from HIN transcripts in aleurone (A), subaleurone (SA), and starchy endosperm (SE) at (A) 12 and (B) $\geq 20$ dap. HIN transcripts in (C) A, (D) SA, and (E) SE at 12 and $\geq 20$ dap. For statistical analyses, we performed a Student's $t$-test. Bars represent standard deviation. Note the indicated $p$-values.

allowing a reliable identification and quantification of HINa, HINb1, and HINb2. To analyze the HIN transcript as well as the protein abundance in more detail, we performed spatio-temporal RT-qPCR as well as proteomics to quantify the transcript and protein levels at 12 and $\geq 20$ dap. At both 12 and $\geq 20$ dap, the amounts of HIN transcripts and HIN proteins were higher in starchy endosperm than in aleurone and subaleurone. However, $\mathrm{HINb}$ transcripts were highly abundant in subaleurone at 12 dap where no HIN proteins could be detected. Additionally, whereas $\mathrm{HINa}$ transcripts increased significantly in subaleurone between 12 and $\geq 20$ dap, there is no significant difference between the final amount of $\mathrm{HINa}$ and $\mathrm{HINb}$ transcripts in subaleurone at $\geq 20$ dap. At the protein level, only one peptide of $\mathrm{HINa}$ could be detected in subaleurone at $\geq 20$ dap, indicating that HINa is more abundant than HINbs. These results point to an absence of the correlation between the HIN transcripts and HIN protein abundances in the subaleurone (Figure 8). This difference between transcript and protein levels was already described and discussed during maize endosperm development (Walley et al., 2013). In this context, it would be interesting to assess the stability and the translation rate of the HIN transcripts in all tissues, especially in subaleurone. Similarly, a comparative study between the different HIN protein stabilities could be conducted.

The presence of HINs was studied previously on molecular level but no information is available about their subcellular localization. PINs in wheat as well as TRPs in oat are known to accumulate at starch granules in late-developed and mature cells (Dubreil et al., 1998; Mohammadi et al., 2006). The used polyclonal anti-PINa recognized both PINs in immunostaining studies in aleurone and subaleurone, whereas monoclonal antiPINa antibody only gave signals in the starchy endosperm, suggesting a different spatial distribution (Dubreil et al., 1998). Both PINs PINa and PINb were detected previously at PBs in developing wheat endosperm, describing the same trafficking 

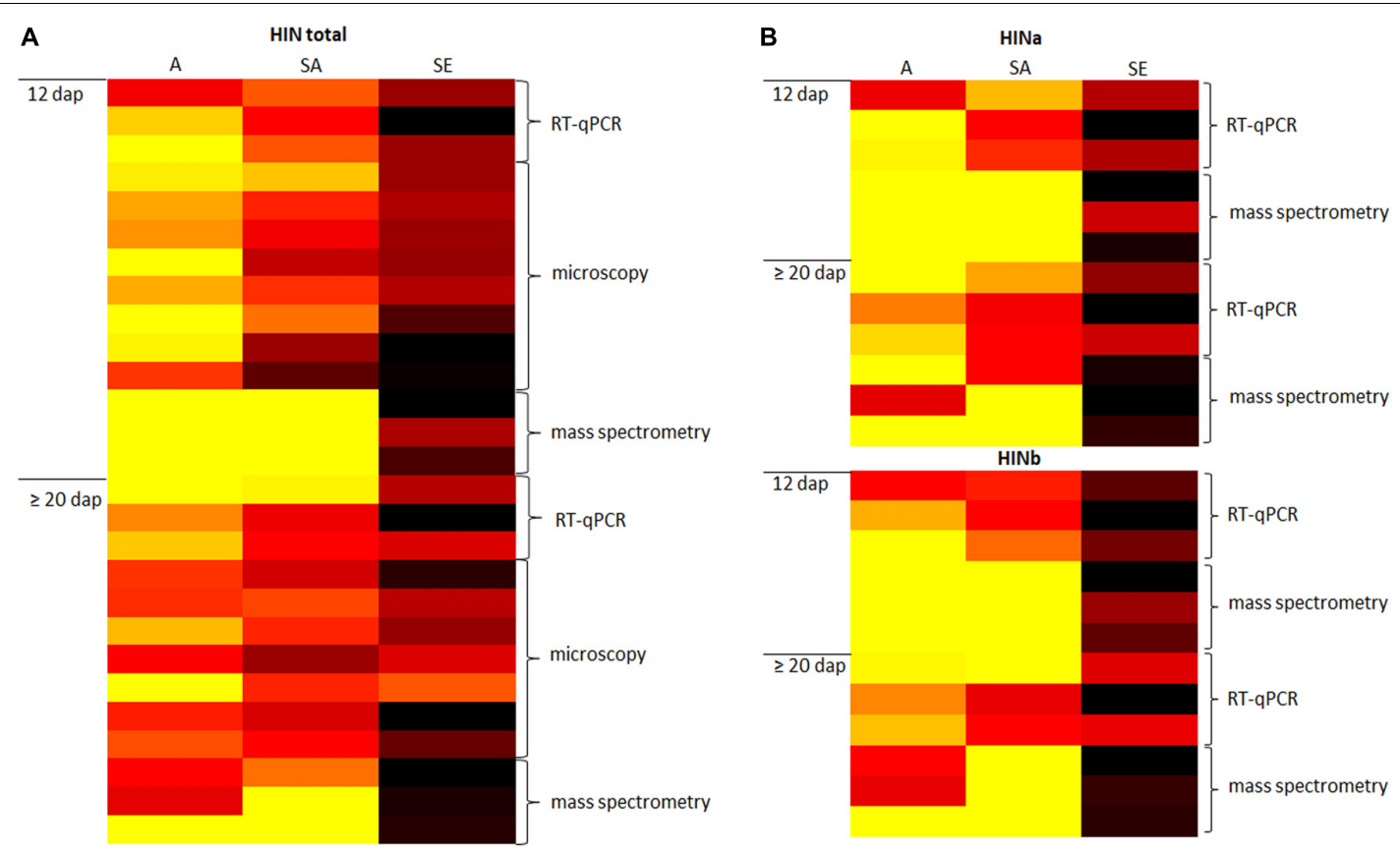

lowest

FIGURE 7 | Data-matrix heat map illustrating the correlation of RT-qPCR, microscopy, and mass spectrometry data of the spatio-temporal expression abundance of HINs in developing barley endosperm. (A) Data-matrix heat map of all HINs. RT-qPCR cluster represents the normalization results $(n=3)$, the microscopy data represent the area calculation ( $n=8$ for 12 dap; $n=7$ for $\geq 20$ dap), and the mass spectrometry data represent the total amount of HINs ( $n=3$ ). (B) Data-matrix heat map of HINa and HINb. RT-qPCR cluster represents the normalization results $(n=3)$ of HINa and HINb, and the mass spectrometry data represent the total amount of $\mathrm{HINa}(n=3)$ and $\mathrm{HINb}(n=3)$. Heat map was prepared by the Software Microsoft Excel. Scale: yellow = smallest value; red = 50\% quantil; black = highest value.

pathway as prolamins from the endoplasmic reticulum (ER) to PBs (Lesage et al., 2011; Ibl and Stoger, 2012). As PINs show a close structural relation to $2 \mathrm{~S}$ storage protein, it was suggested that PINs could be $2 \mathrm{~S}$-like storage proteins in wheat and possibly interact with prolamins via their tryptophan-rich domain (Lesage et al., 2011). This result points to a link between endosperm texture and storage protein aggregation. Here, we showed that the localization of HIN proteins is spatio-temporally regulated within the barley endosperm as we found different strong fluorescent signals at PBs and at the periphery of starch granules at 12 and $\geq 20$ dap. At 12 and $\geq 20$ dap, we detected HINs mainly in the subaleurone and in the starchy endosperm at PBs and additionally at the periphery of starch granules. At $\geq 20$ dap, the fluorescent signal around the starch granules increased while the signal in the PB remained stable in the starchy endosperm. An additional signal was visible in the aleurone at $\geq 20$ dap, indicating the presence of additional HIN proteins at $\geq 20$ dap in the aleurone. As we have been working with polyclonal anti$\mathrm{PINa} / \mathrm{b}$, we were not able to discriminate between HINa and $\mathrm{HINb}$ proteins in these localization studies.

Different final localizations of HINs may include the involvement of diverse protein trafficking pathways. However, the precise trafficking route of HINs to their final destinations is still unclear. The cereal endosperm contains specialized organelles for the accumulation of SSP, which are ultimately deposited either within PB derived from the ER, or in PSVs (reviewed in Arcalis et al., 2014). Besides dense vesicles, multivesicular bodies (MVBs) thereby play a critical role in post-Golgi transport of SSP toward the vacuole (reviewed in Ibl and Stoger, 2012). Additionally, an unusual autophagylike mechanism for the vacuolar delivery of prolamins was discussed in maize aleurone cells where prolamin-containing compartments are eventually fusing with MVBs to form hybrid prevacuolar compartments (Reyes et al., 2011). Obviously, SSP transport routes depend on the cereal species, endosperm tissue layer, and developmental timepoint (Ibl and Stoger, 2012; Zheng and Wang, 2014). Recently, we showed that the involvement of HvVPS60a, a component of the ESCRT machinery coordinating the sorting of ubiquitinated membrane proteins into intraluminal vesicles (ILVs), is depending on the cell layer (Winter and Hauser, 2006; Hilscher et al., 2015). Additionally, OsVPS22 (ESCRT-II) is supposed to be required for seedling viability and grain filling in rice as the $v p s 22$ mutant in rice endosperm showed a chalky endosperm (Zhang et al., 2013). We identified HINs as putative weak interaction partners of an ESCRT-III protein by co-immunoprecipitation and yeast two-hybrid studies (manuscript in preparation). These results let us speculate about the involvement of the ESCRT machinery in the targeting of HINs in barley endosperm development. Further investigations are needed 

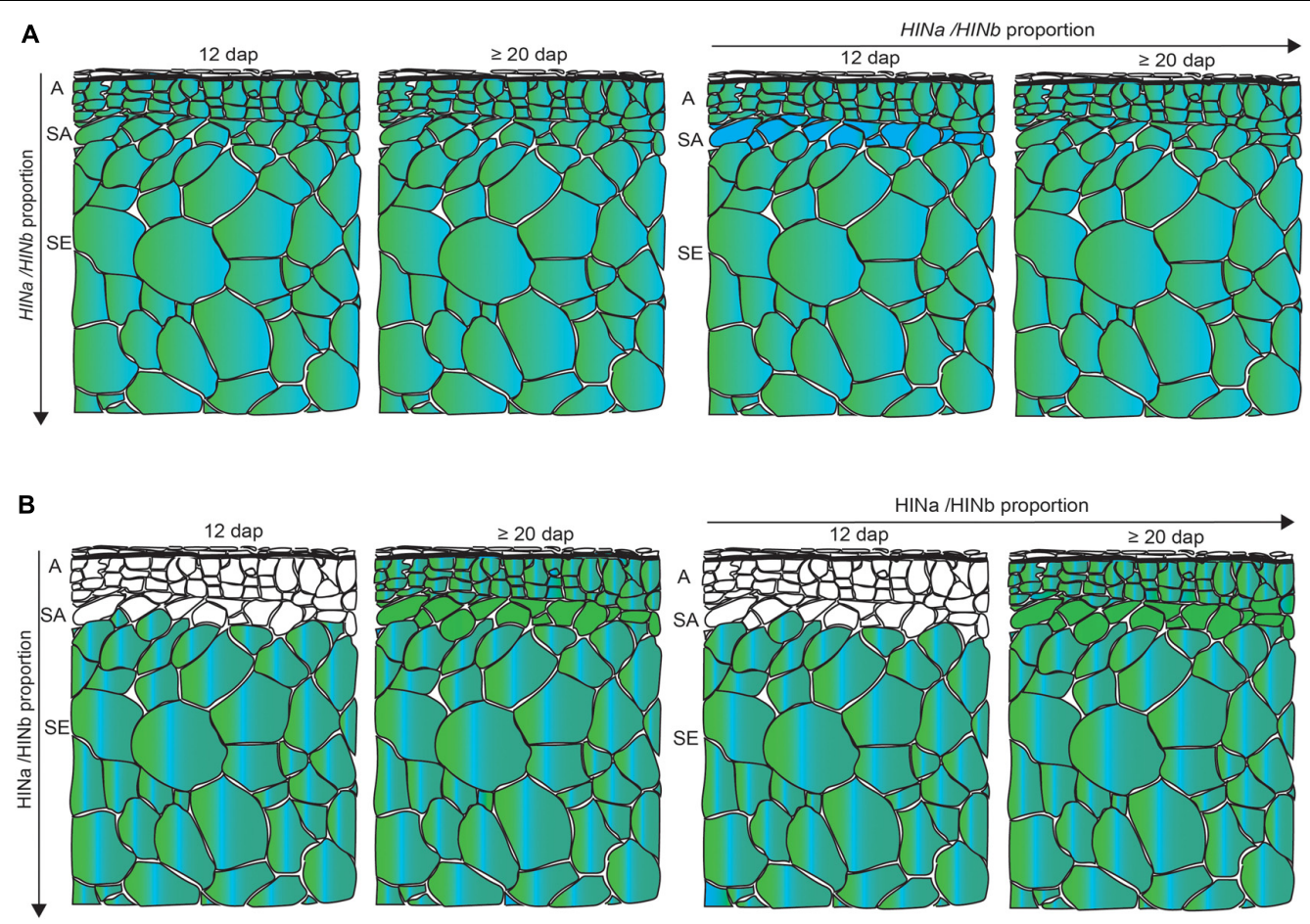

FIGURE 8 | Schematic overview of the spatio-temporal HIN transcript and HIN protein abundance in developing barley endosperm. (A) HINa/HINb transcripts proportion in all tissues between 12 and $\geq 20$ dap and between aleurone (A), subaleurone (SA), and starchy endosperm (SE) between 12 and $\geq 20$ dap.

(B) $\mathrm{HINa} / \mathrm{HINb}$ protein abundance proportion in all tissues between 12 and $\geq 20$ dap and between $\mathrm{A}, \mathrm{SA}$, and SE between 12 and $\geq 20$ dap. Color code: green, $\mathrm{HINa}$; bright blue, HINb1; and turquoise, HINb2. White cells represent no detected HIN proteins. Note the differences in the SA layer. Figure was drawn by the software program Adobe Illustrator CC 2015.

in barley endosperm to follow the trafficking dynamic of HINs.

Finally, we propose another explanation for the different localization of HIN proteins observed in our immunofluorescent microscopy results: indeed, the identified peptides indicate that trypsin digestion can release cytotoxic peptides. The cytotoxicity of tryptophan-rich peptides has been studied and it is suggested that the cytotoxic mechanism seems to affect membrane permeability (Alfred et al., 2013; Haney et al., 2013; Ishida et al., 2016; Shagaghi et al., 2016). Is it legitimate to interrogate the cytotoxicity of tryptophan-rich peptides for cereal cells? And if yes, what is the scavenging process? In this context, it is worth to mention that PINs are still present in baked and stored food products (Capparelli et al., 2005; Pauly et al., 2013a,b).

Indeed, the question arises how the spatio-temporal regulated HIN protein abundance and HINs' localization affect the food quality? The differences in the endosperm texture related to hardness have an impact on the properties and quality of flour: soft wheat flour is used for producing cake and cookies hard wheat flour for bread (summarized in Pauly et al., 2013a,b). However, it is still unclear how PINs behave in the cerealbased end-products, as most of the lipid binding capacity and foaming properties have been demonstrated in vitro. Nevertheless, previous studies showed that the amount of PINb bound to starch in soft wheat is twofold higher than that of HINa and HINb in barley cultivars (Gazza et al., 2008;
Galassi et al., 2012). However, Galassi et al. (2012) suggested that the ratio between $\mathrm{HINb} / \mathrm{HINa}$ is important for the hard texture of the barley grain as the $\mathrm{PINb} / \mathrm{PINa}$ ratio in softtextured wheat is threefold higher than the $\mathrm{HINb} / \mathrm{HINa}$ ration in barley. It was further speculated that the poor interaction of $\mathrm{HINa}$ and HINb during their deposition on the starch surface is responsible for the different barley kernel texture (Galassi et al., 2012). Our results bring evidence that at $\geq 20$ dap, the protein abundance differs significantly between HINa and HINb1/HINb2 in starchy endosperm but not at 12 dap. Additionally, HINa transcripts as well as HINa proteins accumulate specifically in the subaleurone at $\geq 20 \mathrm{dap}$, pointing to a different ration of $\mathrm{HINb} / \mathrm{HINa}$ in this tissue and thus subsequently indicating a putative additional function of HINa in subaleurone. Therefore, it would be necessary to study the localization with specific antibodies for HINa and $\mathrm{HINb}$ to analyze the $\mathrm{HINb} / \mathrm{HINa}$ ratio on subcellular level and to elucidate the spatio-temporal protein trafficking pathway necessary for their final destinations in barley endosperm.

\section{CONCLUSION}

In this study, we used RT-qPCR, proteomics, and microscopy to unravel the spatio-temporal HIN expression and subcellular localization alterations in developing GP endosperm. To enable 
RT-qPCR in the cultivar GP, we characterized RGs for spatiotemporal normalization that can be used to carry out gene transcript analyses in developing GP endosperm. Based on our correlating results, we show that the expression abundance of $\mathrm{HINs}$ and the ration of $\mathrm{HINb} / \mathrm{HINa}$ is tissue dependent during GP endosperm development. These findings may point to the necessity to study cereal endosperms tissue specific to improve plant crop engineering in terms of grain kernel texture modifications and end-product quality. Therefore, a good transcriptomic and proteomic map will help to understand the molecular mechanism behind these rearrangements. This will subsequently enable to specifically manipulate the $\mathrm{HINb} / \mathrm{HINa}$ ratio in different tissues during development stages that could result in hard or soft barley kernel texture and thus influences the end-product quality.

\section{AUTHOR CONTRIBUTIONS}

VI acquired funding. VI and VR conceived and designed the experiments. AS, VR, and VI wrote the manuscript. AS performed the RT-qPCR analyses. VR did the MS analyses. P-JR performed the western blots. SR did the embedding. MW performed the semi-thin sectioning. ES and WW contributed toward the data analyses. All authors read and approved the final version of the manuscript for publication.

\section{FUNDING}

This work was supported by the Austrian Science Fund (FWF Project P29454-B22 and P 29303-B22).

\section{ACKNOWLEDGMENTS}

We thank Dr. Julia Hilscher and Christiane Schwartz for RTqPCR support, Dr. Karl Schedle from the Institute of Animal Nutrition, Livestock Products, and Nutrition Physiology (TTE), University of Natural Resources and Life Sciences, Vienna, for sharing the RNA lab. We thank the BOKU-VIBT ImagingCenter and Dr. Monika Debreczeny for help with LMD. We acknowledge Dr. Illimar Altosaar, University of Ottawa, Canada, for kindly providing anti-PINa/b antisera. We thank Laura Ullrich, Dr. Michele Grieco, Dr. Cornelia Konlechner, Dr. Alois Schweighofer, and Dr. Ingeborg Lang for critical reading of the manuscript.

\section{SUPPLEMENTARY MATERIAL}

The Supplementary Material for this article can be found online at: https://www.frontiersin.org/articles/10.3389/fpls.2018.00775/ full\#supplementary-material

FIGURE S1 | Schematic workflow of our multi-disciplinary approach as described in Section "Materials and Methods."

FIGURE S2 | RT-qPCR reaction quality control and amplification efficiency of 10 candidate RGs for RT-qPCR in the barley cultivar GP during endosperm development. (A) Detection of cDNA of all RGs. M: DNA ladder $100 \mathrm{bp}$. Primer pairs are indicated by an asterisk. (B) Quantification of RNA extracted from seeds at $6,10,12$, and $\geq 20$ dap. RNA integrity was controlled photometrically (260/280). (C) Results from standard curves of the selected candidate RGs: slope, $R^{2}$ value, reaction efficiency (RE), and amplification factor (AF). (D) Relative quantification of all RGs from the whole seed harvested at $6,10,12$, and $\geq 20$ dap. (E) Coefficient of variation (CV) of all RGs. (F) Average expression stability (M) of all RGs from the whole seed harvested at $6,10,12$, and $\geq 20$ dap calculated by geNorm. (G) Calculation of the pairwise variation by geNorm.

FIGURE S3 | RT-qPCR reaction quality control and amplification efficiency of 10 candidate RGs for spatio-temporal RT-qPCR during GP endosperm development. (A) Quantification of RNA extracted from aleurone, subaleurone, and starchy endosperm at 12 and $\geq 20$ dap. RNA integrity was controlled photometrically (260/280). (B) Results from standard curves of the selected candidate RGs: slope, $R^{2}$ value, reaction efficiency (RE), and amplification factor (AF). (C) Relative quantification of all RGs from all tissues at 12 and $\geq 20$ dap. (D) Relative quantification of all RGs from and from aleurone (A), subaleurone (SA), and starchy endosperm (SE) at 12 and $\geq 20$ dap.

FIGURE S4 | RT-qPCR reaction quality control and amplification efficiency of 10 candidate RGs for RT-qPCR in GP endosperm development at 12 and $\geq 20$ dap for all tissues. (A) Coefficient of variation (CV) of all RGs for all tissues at 12 and $\geq 20$ dap. (B) Average expression stability ( $M$ ) of all RGs from the all tissues at 12 dap calculated by geNorm. Note the calculation of the pairwise variation by geNorm. (C) Average expression stability ( $M$ ) of all RGs from the all tissues at $\geq 20$ dap calculated by geNorm. Note the calculation of the pairwise variation by geNorm.

FIGURE S5 | RT-qPCR reaction quality control and amplification efficiency of 10 candidate RGs for RT-qPCR in barley endosperm for aleurone (A), subaleurone (SA), and starchy endosperm (SE) at 12 and $\geq 20$ dap. (A) Coefficient of variation (CV) of all RGs for $A$ (12 dap, $\geq 20$ dap), SA (12 and $\geq 20$ dap), and SE (12 and $\geq 20$ dap). Average expression stability ( $M$ ) of all $R G$ for (B) $A$ (12 and $\geq 20$ dap), (C) SA (12 and $\geq 20$ dap), and (D) SE (12 and $\geq 20$ dap). Note the calculation of the pairwise variation by geNorm.

FIGURE S6 | RT-qPCR reaction quality control and amplification efficiency of the HIN genes by RT-qPCR in barley endosperm. (A) CDNA amplification of HINa and HINb. M: DNA ladder $100 \mathrm{bp}$. (B) Results from standard curves of HINa and HINb: slope, $R^{2}$ value, reaction efficiency (RE), and amplification factor (AF).

FIGURE S7 | Bar graph describes the average over three biological replicates of the normalized transcripts from HINa and HINb at 6, 10, 12, and $\geq 20$ dap with the most unstable RG (GRP). Bars represent standard deviation.

FIGURE S8 | Identification and molecular characterization of $\mathrm{HINa}$, $\mathrm{HINb}$, and $\mathrm{HINb2}$. (A) Identified peptides related to $\mathrm{HINa}$ (green), HINb1 (bright blue), and HINb2 (turquoise). (B) Protein alignment of the tryptophanins (oat), puroindolines (wheat), and hordoindolines (barley). Note the signal peptide, propeptide, the tryptophan-rich side, and the cysteine amino acids (orange box). Alignment was performed by MEGA7.0.21 (Kumar et al., 2016) and visualized by GenDoc (Nicholas and Nicholas, 1997). Conserved percentage is shown as the following: black $100 \%$, dark gray $80 \%$. (C) Western blot of extracted proteins isolated at 6 , 12 , and $\geq 20$ dap incubated with anit-PINa/b. Note the increase of the HIN protein abundance during barley endosperm development.

FIGURE S9 | Negative control of microscopic studies using secondary antibody Alexa488 ${ }^{\circledR}$. Scale $=100 \mu \mathrm{m}$.

FIGURE S10 | Quantification of the fluorescent signal of anti-PINa/PINb. (A) We used ImageJ to mark areas in aleurone (A), subaleurone (SA), and starchy endosperm (SE) at 12 and $\geq 20$ dap for the mean intensity identification. (B) Bar blots of the fluorescent intensity of anti-PINa/b in all tissues at 12 and $\geq 20$ dap and in A, SA, and SE at 12 and $\geq 20$ dap. Scale is $100 \mu \mathrm{m}$. Areas ( $n=8$ for 12 dap; $n=7$ for $\geq 20$ dap) were quantified for $A, S A$, and SE from at least two slides with three sections. For statistical analyses, we performed a Student's $t$-test. Bars represent standard deviation. Note the indicated $p$-values.

TABLE S1 | Selected RGs are listed by their gene number, gene symbol, gene product function, gene ID (Unigene), primer sequence, amplicon length, 
corresponding mRNA sequence, corresponding protein (Uniprot) entry, and the identified protein in our MS data.

TABLE S2 | Ranking of the RGs for spatio-temporal RT-qPCR analyses in the barley cultivar Golden Promise during caryopsis development.

\section{REFERENCES}

Alfred, R. L., Palombo, E. A., Panozzo, J. F., and Bhave, M. (2013). The antimicrobial domains of wheat puroindolines are cell-penetrating peptides with possible intracellular mechanisms of action. PLoS One 8:e75488. doi: 10.1371/journal.pone.0075488

Andersen, C. L., Jensen, J. L., and Orntoft, T. F. (2004). Normalization of real-time quantitative reverse transcription-PCR data: a model-based variance estimation approach to identify genes suited for normalization, applied to bladder and colon cancer data sets. Cancer Res. 64, 5245-5250. doi: 10.1158/0008-5472. CAN-04-0496

Arcalis, E., Ibl, V., Peters, J., Melnik, S., and Stoger, E. (2014). The dynamic behavior of storage organelles in developing cereal seeds and its impact on the production of recombinant proteins. Front. Plant Sci. 5:439. doi: 10.3389/fpls.2014. 00439

Bamforth, C. W. (1985). The Foaming Properties of Beer. J. Instit. Brew. 91, 370-383. doi: 10.1002/j.2050-0416.1985.tb04359.x

Bhave, M., and Morris, C. F. (2008). Molecular genetics of puroindolines and related genes: regulation of expression, membrane binding properties and applications. Plant Mol. Biol. 66, 221-231. doi: 10.1007/s11103-0079264-6

Blochet, J. E., Chevalier, C., Forest, E., Pebay-Peyroula, E., Gautier, M. F., Joudrier, P., et al. (1993). Complete amino acid sequence of puroindoline, a new basic and cystine-rich protein with a unique tryptophan-rich domain, isolated from wheat endosperm by Triton X-114 phase partitioning. FEBS Lett. 329, 336-340. doi: 10.1016/0014-5793(93)80249-T

Brennan, C. S., Harris, N., Smith, D., and Shewry, P. R. (1996). Structural differences in the mature endosperms of good and poor malting barley cultivars. J. Cereal Sci. 24, 171-177. doi: 10.1006/jcrs.1996.0050

Bustin, S. A., Benes, V., Garson, J. A., Hellemans, J., Huggett, J., Kubista, M., et al. (2009). The MIQE Guidelines: minimum information for publication of quantitative real-time PCR experiments. Clin. Chem. 55, 611-622. doi: 10.1373/ clinchem.2008.112797

Capparelli, R., Amoroso, M. G., Palumbo, D., Iannaccone, M., Faleri, C., and Cresti, M. (2005). Two plant puroindolines colocalize in wheat seed and in vitro synergistically fight against pathogens. Plant Mol. Biol. 58, 857-867. doi: 10.1007/s11103-005-8270-9

Carlemalm, E., Villiger, W., Hobot, J. A., Acetarin, J. D., and Kellenberger, E. (1985). Low temperature embedding with Lowicryl resins: two new formulations and some applications. J. Microsc. 140(Pt 1), 55-63. doi: 10.1111/j.1365-2818.1985.tb02660.x

Clark, D. C., Wilde, P. J., and Marion, D. (1994). The protection of beer foam against lipid-induced destabilization. J. Instit. Brew. 100, 23-25. doi: 10.1002/ j.2050-0416.1994.tb00806.x

Cox, J., Hein, M. Y., Luber, C. A., Paron, I., Nagaraj, N., and Mann, M. (2014). Accurate proteome-wide label-free quantification by delayed normalization and maximal peptide ratio extraction, termed MaxLFQ. Mol. Cell. Proteomics 13, 2513--2526. doi: 10.1074/mcp.M113.031591

Cox, J., and Mann, M. (2008). MaxQuant enables high peptide identification rates, individualized p.p.b.-range mass accuracies and proteome-wide protein quantification. Nat. Biotechnol. 26, 1367-1372. doi: 10.1038/ nbt. 1511

Cox, J., Neuhauser, N., Michalski, A., Scheltema, R. A., Olsen, J. V., and Mann, M. (2011). Andromeda: a peptide search engine integrated into the MaxQuant environment. J. Proteome Res. 10, 1794-1805. doi: 10.1021/pr101065j

Darlington, H. F., Rouster, J., Hoffmann, L., Halford, N. G., Shewry, P. R., and Simpson, D. J. (2001). Identification and molecular characterisation of hordoindolines from barley grain. Plant Mol. Biol. 47, 785-794. doi: 10.1023/A: 1013691530675

Deutsch, E. W., Csordas, A., Sun, Z., Jarnuczak, A., Perez-Riverol, Y., Ternent, T., et al. (2017). The ProteomeXchange consortium in 2017: supporting the
TABLE S3 | HINa, HINb1, and HINb2 are listed by their gene symbol, gene product function, cDNA entry (Uniprot), primer sequence, amplicon length, corresponding protein (Uniprot) entry, and the identified protein in our MS data.

TABLE S4 | MS data.

cultural change in proteomics public data deposition. Nucleic Acids Res. 45, D1100-D1106. doi: 10.1093/nar/gkw936

Dubreil, L. (1997). Interaction of puroindolines with wheat flour polar lipids determines their foaming properties. J. Agric. Food Chem. 45, 108-116. doi: 10.1021/jf960402j

Dubreil, L., Gaborit, T., Bouchet, B., Gallant, D. J., Broekaert, W. F., Quillien, L., et al. (1998). Spatial and temporal distribution of the major isoforms of puroindolines (puroindoline-a and puroindoline-b) and non specific lipid transfer protein (ns-LTPle(1)) of Triticum aestivum seeds. Relationships with their in vitro antifungal properties. Plant Sci. 138, 121-135. doi: 10.1016/S01689452(98)00121-6

Faccioli, P., Ciceri, G. P., Provero, P., Stanca, A. M., Morcia, C., and Terzi, V. (2007). A combined strategy of \&quot;in silico\&quot; transcriptome analysis and web search engine optimization allows an agile identification of reference genes suitable for normalization in gene expression studies. Plant Mol. Biol. 63, 679-688. doi: 10.1007/s11103-0069116-9

Galassi, E., Gazzelloni, G., Taddei, F., Muccilli, V., Gazza, L., and Pogna, N. (2012). Kernel texture and hordoindoline patterns in barley (Hordeum vulgare). Mol. Breed. 30, 1551-1562. doi: 10.1007/s11032-012-9738-3

Gautier, M. F., Aleman, M. E., Guirao, A., Marion, D., and Joudrier, P. (1994). Triticum aestivum puroindolines, two basic cystine-rich seed proteins: cDNA sequence analysis and developmental gene expression. Plant Mol. Biol. 25, 43-57. doi: 10.1007/BF00024197

Gazza, L., Taddei, F., Corbellini, M., Cacciatori, P., and Pogna, N. E. (2008). Genetic and environmental factors affecting grain texture in common wheat. J. Cereal Sci. 47, 52-58. doi: 10.1016/j.jcs.2007.01.004

Giroux, M. J., and Morris, C. F. (1998). Wheat grain hardness results from highly conserved mutations in the friabilin components puroindoline a and b. Proc. Natl. Acad. Sci. U.S.A. 95, 6262-6266. doi: 10.1073/pnas.95. 11.6262

Greenwell, P., and Schofield, J. D. (1986). A starch granule protein associated with endosperm softness in wheat. Cereal Chem. 63, 379-380.

Gupta, M., Abu-Ghannam, N., and Gallaghar, E. (2010). Barley for brewing: characteristic changes during malting, brewing and applications of its byproducts. Compr. Rev. Food Sci. Food Saf. 9, 318-328. doi: 10.1111/j.1541-4337. 2010.00112.x

Haney, E. F., Petersen, A. P., Lau, C. K., Jing, W., Storey, D. G., and Vogel, H. J. (2013). Mechanism of action of puroindoline derived tryptophan-rich antimicrobial peptides. Biochim. Biophys. Acta 1828, 1802-1813. doi: 10.1016/j. bbamem.2013.03.023

Hiei, Y., Ishida, Y., and Komari, T. (2014). Progress of cereal transformation technology mediated by Agrobacterium tumefaciens. Front. Plant Sci. 5:628. doi: $10.3389 /$ fpls.2014.00628

Hilscher, J., Kapusi, E., Stoger, E., and Ibl, V. (2015). Cell layer-specific distribution of transiently expressed barley ESCRT-III component HvVPS60 in developing barley endosperm. Protoplasma 253, 137-153. doi: 10.1007/s00709-0150798-1

Holzberg, S., Brosio, P., Gross, C., and Pogue, G. P. (2002). Barley stripe mosaic virus-induced gene silencing in a monocot plant. Plant J. 30, 315-327. doi: 10.1046/j.1365-313X.2002.01291.x

Huggett, J., Dheda, K., Bustin, S., and Zumla, A. (2005). Real-time RT-PCR normalisation; strategies and considerations. Genes Immun. 6, 279-284. doi: 10.1038/sj.gene.6364190

Ibl, V., Kapusi, E., Arcalis, E., Kawagoe, Y., and Stoger, E. (2014). Fusion, rupture, and degeneration: the fate of in vivo-labelled PSVs in developing barley endosperm. J. Exp. Bot. 65, 3249-3261. doi: 10.1093/jxb/ eru175

Ibl, V., and Stoger, E. (2012). The formation, function and fate of protein storage compartments in seeds. Protoplasma 249, 379-392. doi: 10.1007/s00709-0110288-z 
Ibl, V., and Stoger, E. (2014). Live cell imaging during germination reveals dynamic tubular structures derived from protein storage vacuoles of barley aleurone cells. Plants 3, 442-457. doi: 10.3390/plants3030442

Ishida, H., Nguyen, L. T., Gopal, R., Aizawa, T., and Vogel, H. J. (2016). Overexpression of antimicrobial, anticancer, and transmembrane peptides in Escherichia coli through a calmodulin-peptide fusion system. J. Am. Chem. Soc. 138, 11318-11326. doi: 10.1021/jacs.6b06781

Koressaar, T., Lepamets, M., Kaplinski, L., Raime, K., Andreson, R., and Remm, M. (2018). Primer3_masker: integrating masking of template sequence with primer design software. Bioinformatics doi: 10.1093/bioinformatics/bty036 [Epub ahead of print].

Kozera, B., and Rapacz, M. (2013). Reference genes in real-time PCR. J. Appl. Genet. 54, 391-406. doi: 10.1007/s13353-013-0173-x

Krishnamurthy, K., Balconi, C., Sherwood, J. E., and Giroux, M. J. (2001). Wheat puroindolines enhance fungal disease resistance in transgenic rice. Mol. Plant Microbe Interact. 14, 1255-1260. doi: 10.1094/MPMI.2001.14.10.1255

Krishnamurthy, K., and Giroux, M. J. (2001). Expression of wheat puroindoline genes in transgenic rice enhances grain softness. Nat. Biotechnol. 19, 162-166. doi: $10.1038 / 84435$

Kumar, S., Stecher, G., and Tamura, K. (2016). MEGA7: molecular evolutionary genetics analysis Version 7.0 for bigger datasets. Mol. Biol. Evol. 33, 1870-1874. doi: $10.1093 / \mathrm{molbev} / \mathrm{msw} 054$

Lee, W. S., Hammond-Kosack, K. E., and Kanyuka, K. (2012). Barley stripe mosaic virus-mediated tools for investigating gene function in cereal plants and their pathogens: virus-induced gene silencing, host-mediated gene silencing, and virus-mediated overexpression of heterologous protein. Plant Physiol. 160, 582-590. doi: 10.1104/pp.112.203489

Lesage, V. S., Bouchet, B., Rhazi, L., Elmorjani, K., Branlard, G., and Marion, D. (2011). New insight into puroindoline function inferred from their subcellullar localization in developing hard and soft near-isogenic endosperm and their relationship with polymer size of storage proteins. J. Cereal Sci. 53, 231-238. doi: 10.1016/j.jcs.2011.01.002

Lesage, V. S., Merlino, M., Chambon, C., Bouchet, B., Marion, D., and Branlard, G. (2012). Proteomes of hard and soft near-isogenic wheat lines reveal that kernel hardness is related to the amplification of a stress response during endosperm development. J. Exp. Bot. 63, 1001-1011. doi: 10.1093/jxb/err330

Li, W. L., Huang, L., and Gill, B. S. (2008). Recurrent deletions of Puroindoline genes at the grain Hardness locus in four independent lineages of polyploid wheat. Plant Physiol. 146, 200-212. doi: 10.1104/pp.107.108852

Li, Z. W., and Trick, H. N. (2005). Rapid method for high-quality RNA isolation from seed endosperm containing high levels of starch. Biotechniques 38, 872-876. doi: 10.2144/05386bm05

Ling, D., and Salvaterra, P. M. (2011). Robust RT-qPCR data normalization: validation and selection of internal reference genes during post-experimental data analysis. PLoS One 6:e17762. doi: 10.1371/journal.pone.0017762

Mahalingam, R. (2017). Shotgun proteomics of the barley seed proteome. BMC Genomics 18:44. doi: 10.1186/s12864-016-3408-5

Mohammadi, M., Abbas Zaidi, M., Ochalski, A., Tanchak, M., and Altosaar, I. (2006). Immunodetection and immunolocalization of tryptophanins in oat (Avena sativa L.) seeds. Plant Sci. 172, 579-587. doi: 10.1016/j.plantsci.2006. 11.015

Nicholas, K. B., and Nicholas, H. B. J. (1997). GeneDoc: A Tool for Editing and Annotating Multiple Sequence Alignments. Available at: http://www.psc.edu/ biomed/genedoc

Olsen, O. A. (2001). Endosperm development: cellularization and cell fate specification. Annu. Rev. Plant Physiol. Plant Mol. Biol. 52, 233-267. doi: 10.1146/annurev.arplant.52.1.233

Olsen, O. A. (2004). Nuclear endosperm development in cereals and Arabidopsis thaliana. Plant Cell 16(Suppl.), S214-S227. doi: 10.1105/tpc.017111

Ovesna, J., Kucera, L., Vaculova, K., Strymplova, K., Svobodova, I., and Milella, L. (2012). Validation of the beta-amyl transcription profiling assay and selection of reference genes suited for a RT-qPCR assay in developing barley caryopsis. PLoS One 7:e41886. doi: 10.1371/journal.pone. 0041886

Pauly, A., Pareyt, B., Fierens, E., and Delcour, J. A. (2013a). Wheat (Triticum aestivum L. and T. turgidum L. ssp durum) Kernel hardness: I. current view on the role of puroindolines and polar lipids. Compr. Rev. Food Sci. Food Saf. 12, 413-426. doi: 10.1111/1541-4337.12019
Pauly, A., Pareyt, B., Fierens, E., and Delcour, J. A. (2013b). Wheat (Triticum aestivum L. and T. turgidum L. ssp durum) kernel hardness: II. implications for end-product quality and role of puroindolines therein. Compr. Rev. Food Sci. Food Saf. 12, 427-438. doi: 10.1111/1541-4337.12018

Peukert, M., Thiel, J., Peshev, D., Weschke, W., Van den Ende, W., Mock, H. P., et al. (2014). Spatio-temporal dynamics of fructan metabolism in developing barley grains. Plant Cell 26, 3728-3744. doi: 10.1105/tpc.114.130211

Psota, V., Vejrazka, K., Famera, O., and Hrcka, M. (2007). Relationship between grain hardness and malting quality of barley (Hordeum vulgare L.). J. Instit. Brew. 113, 80-86. doi: 10.1002/j.2050-0416.2007.tb00260.x

Reipert, S., and Wiche, G. (2008). High-pressure freezing and low-temperature fixation of cell monolayers grown on sapphire coverslips. Methods Cell Biol. 88, 165-180. doi: 10.1016/S0091-679X(08)00410-X

Reyes, F. C., Chung, T., Holding, D., Jung, R., Vierstra, R., and Otegui, M. S. (2011). Delivery of prolamins to the protein storage vacuole in maize aleurone cells. Plant Cell 23, 769-784. doi: 10.1105/tpc.110.082156

Roustan, V., Bakhtiari, S., Roustan, P.-J., and Weckwerth, W. (2017). Quantitative in vivo phosphoproteomics reveals reversible signaling processes during nitrogen starvation and recovery in the biofuel model organism Chlamydomonas reinhardtii. Biotechnol. Biofuels 10:280. doi: 10.1186/s13068-017-0949-z

Schindelin, J., Arganda-Carreras, I., Frise, E., Kaynig, V., Longair, M., Pietzsch, T., et al. (2012). Fiji: an open-source platform for biological-image analysis. Nat. Methods 9, 676-682. doi: 10.1038/nmeth.2019

Schneider, C. A., Rasband, W. S., and Eliceiri, K. W. (2012). NIH Image to ImageJ: 25 years of image analysis. Nat. Methods 9, 671-675. doi: 10.1038/nmeth.2089

Shagaghi, N., Alfred, R. L., Clayton, A. H., Palombo, E. A., and Bhave, M. (2016). Anti-biofilm and sporicidal activity of peptides based on wheat puroindoline and barley hordoindoline proteins. J. Pept. Sci. 22, 492-500. doi: 10.1002/psc. 2895

Sourdille, P., Perretant, M. R., Charmet, G., Leroy, P., Gautier, M. F., Joudrier, P., et al. (1996). Linkage between RFLP markers and genes affecting kernel hardness in wheat. Theor. Appl. Genet. 93, 580-586. doi: 10.1007/BF00417951

Takahashi, A., Ikeda, T. M., Takayama, T., and Yanagisawa, T. (2010). A barley Hordoindoline mutation resulted in an increase in grain hardness. Theor. Appl. Genet. 120, 519-526. doi: 10.1007/s00122-009-1172-5

Tanchak, M. A., Schernthaner, J. P., Giband, M., and Altosaar, I. (1998). Tryptophanins: isolation and molecular characterization of oat cDNA clones encoding proteins structurally related to puroindoline and wheat grain softness proteins. Plant Sci. 137, 173-184. doi: 10.1016/S0168-9452(98)00105-8

Terasawa, Y., Rahman, S. M., Takata, K., and Ikeda, T. M. (2012). Distribution of Hordoindoline genes in the genus Hordeum. Theor. Appl. Genet. 124, 143-151. doi: 10.1007/s00122-011-1693-6

Tyanova, S., Temu, T., Carlson, A., Sinitcyn, P., Mann, M., and Cox, J. (2015). Visualization of LC-MS/MS proteomics data in MaxQuant. Proteomics 15, 1453-1456. doi: 10.1002/pmic.201400449

Tyanova, S., Temu, T., Sinitcyn, P., Carlson, A., Hein, M. Y., Geiger, T., et al. (2016). The Perseus computational platform for comprehensive analysis of (prote)omics data. Nat. Methods 13, 731-740. doi: 10.1038/Nmeth.3901

Untergasser, A., Nijveen, H., Rao, X., Bisseling, T., Geurts, R., and Leunissen, J. A. (2007). Primer3Plus, an enhanced web interface to Primer3. Nucleic Acids Res. 35, W71-W74. doi: 10.1093/nar/gkm306

Vandesompele, J., De Preter, K., Pattyn, F., Poppe, B., Van Roy, N., De Paepe, A., et al. (2002). Accurate normalization of real-time quantitative RT-PCR data by geometric averaging of multiple internal control genes. Genome Biol. 3:RESEARCH0034. doi: 10.1186/gb-2002-3-7-research0034

Vizcaino, J. A., Csordas, A., del-Toro, N., Dianes, J. A., Griss, J., Lavidas, I., et al. (2016). 2016 update of the PRIDE database and its related tools. Nucleic Acids Res. 44, D447-D456. doi: 10.1093/nar/gkv1145

Wall, M. L., Wheeler, H. L., Huebsch, M. P., Smith, J. C., Figeys, D., and Altosaar, I. (2010). The tryptophan-rich domain of puroindoline is directly associated with the starch granule surface as judged by tryptic shaving and mass spectrometry. J. Cereal Sci. 52, 115-120. doi: 10.1016/j.jcs.2010.04.002

Walley, J. W., Shen, Z., Sartor, R., Wu, K. J., Osborn, J., Smith, L. G., et al. (2013). Reconstruction of protein networks from an atlas of maize seed proteotypes. Proc. Natl. Acad. Sci. U.S.A. 110, E4808-E4817. doi: 10.1073/pnas.1319113110

Winter, V., and Hauser, M. T. (2006). Exploring the ESCRTing machinery in eukaryotes. Trends Plant Sci. 11, 115-123. doi: 10.1016/j.tplants.2006.01.008 
Young, T. E., and Gallie, D. R. (2000). Programmed cell death during endosperm development. Plant Mol. Biol. 44, 283-301. doi: 10.1023/A:10265884 08152

Zhang, X. Q., Hou, P., Zhu, H. T., Li, G. D., Liu, X. G., and Xie, X. M. (2013). Knockout of the VPS22 component of the ESCRT-II complex in rice (Oryza sativa L.) causes chalky endosperm and early seedling lethality. Mol. Biol. Rep. 40, 3475-3481. doi: 10.1007/s11033-0122422-1

Zheng, Y., and Wang, Z. (2014). Protein accumulation in aleurone cells, subaleurone cells and the center starch endosperm of cereals. Plant Cell Rep. 33, 1607-1615. doi: 10.1007/s00299-014-1651-2
Conflict of Interest Statement: The authors declare that the research was conducted in the absence of any commercial or financial relationships that could be construed as a potential conflict of interest.

Copyright (c) 2018 Shabrangy, Roustan, Reipert, Weidinger, Roustan, Stoger, Weckwerth and Ibl. This is an open-access article distributed under the terms of the Creative Commons Attribution License (CC BY). The use, distribution or reproduction in other forums is permitted, provided the original author(s) and the copyright owner are credited and that the original publication in this journal is cited, in accordance with accepted academic practice. No use, distribution or reproduction is permitted which does not comply with these terms. 